\title{
Analysis of blow-ups for the double obstacle problem in dimension two
}

\author{
Gohar Aleksanyan *
}

September 27, 2018

\begin{abstract}
In this article we study a normalised double obstacle problem with polynomial obstacles $p^{1} \leq p^{2}$ under the assumption that $p^{1}(x)=p^{2}(x)$ iff $x=0$. In dimension two we give a complete characterisation of blow-up solutions depending on the coefficients of the polynomials $p^{1}, p^{2}$. In particular, we see that there exists a new type of blow-ups, that we call double-cone solutions since the coincidence sets $\left\{u=p^{1}\right\}$ and $\left\{u=p^{2}\right\}$ are cones with a common vertex.

We prove the uniqueness of blow-up limits, and analyse the regularity of the free boundary in dimension two. In particular we show that if the solution to the double obstacle problem has a double-cone blow-up limit at the origin, then locally the free boundary consists of four $C^{1, \gamma}$-curves, meeting at the origin.

In the end we give an example of a three-dimensional double-cone solution.
\end{abstract}

\section{Contents}

1 Introduction

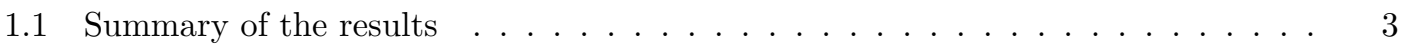

2 Weiss' energy functional for the double obstacle problem 4

3 Characterisation of blow-ups in $\mathbb{R}^{2}$

3.1 Examples . . . . . . . . . . . . . . . . . . . . . . 7

3.2 Double-cone solutions . . . . . . . . . . . . . . . . . . . . . 9

3.3 Halfspace solutions . . . . . . . . . . . . . . . . . . . . 15

4 Uniqueness of blow-ups, Case 1

5 Uniqueness of blow-ups, Case 2

6 Uniqueness of blow-ups, Case 3

7 An example of a double-cone solution in $\mathbb{R}^{3}$

7.1 Solutions symmetric with respect to the $z$-axes $\ldots \ldots \ldots \ldots . \ldots \ldots$

References 35

${ }^{*}$ Current affiliation: Department of Mathematics, University of Duisburg Essen, Thea-Leymann-Strasse 9, 45127 Essen, Germany. E-mail: gohar.aleksanyan@uni-due.de 


\section{Introduction}

Let $\Omega$ be a bounded open set in $\mathbb{R}^{n}$ with smooth boundary. The solution to the double obstacle problem in $\Omega$ is the minimiser of the functional

$$
J(v)=\int_{\Omega}|\nabla v(x)|^{2} d x
$$

over functions $v \in W^{1,2}(\Omega), \psi^{1} \leq v \leq \psi^{2}$, satisfying the boundary condition $v=g$ on $\partial \Omega$. For the problem to be well defined we assume that $\psi^{1} \leq \psi^{2}$ in $\Omega$ and $\psi^{1} \leq g \leq \psi^{2}$ on $\partial \Omega$. The functions $\psi^{1}$ and $\psi^{2}$ are called respectively the lower and the upper obstacles.

If $\psi^{1}<\psi^{2}$ then the problem reduces locally to a single obstacle problem. Therefore we are interested in the case when

$$
\Lambda:=\left\{x \in \Omega: \psi^{1}(x)=\psi^{2}(x)\right\} \neq \emptyset .
$$

It is well known that the solution to the double obstacle problem satisfies the following inequalities

$$
\psi^{1} \leq u \leq \psi^{2}, \quad \Delta u \geq 0 \text { if } u>\psi^{1} \text { and } \Delta u \leq 0 \text { if } u<\psi^{2} .
$$

It has been shown that the solution to the double obstacle problem is locally $C^{1,1}$ under the assumption $\psi^{i} \in C^{2}(\Omega)$, see for instance [3, 5]. Therefore we may rewrite (1.2) as

$$
\psi^{1} \leq u \leq \psi^{2} \text { and } \Delta u=\Delta \psi^{1} \chi_{\left\{u=\psi^{1}\right\}}+\Delta \psi^{2} \chi_{\left\{u=\psi^{2}\right\}}-\Delta \psi^{1} \chi_{\left\{\psi^{1}=\psi^{2}\right\}} \text { a.e. }
$$

where $\chi_{A}$ is the characteristic function of a set $A \subset \mathbb{R}^{n}$.

Let us introduce some notations that will be used throughout. Denote by

$$
\Omega_{1}:=\left\{u>\psi^{1}\right\}, \quad \Omega_{2}:=\left\{u<\psi^{2}\right\}, \text { and } \Omega_{12}:=\Omega_{1} \cap \Omega_{2}
$$

then $\Omega=\Omega_{1} \cup \Omega_{2} \cup \Lambda$, where $\Lambda$ is given by (1.1). Let us observe that $u$ is a harmonic function in $\Omega_{12}$, which we call the noncoincidence set. Define the free boundary for the double obstacle problem

$$
\Gamma:=\partial \Omega_{12} \cap \Omega \subset \Gamma_{1} \cup \Gamma_{2} \text {, where } \Gamma_{i}:=\partial \Omega_{i} \cap \Omega, i=1,2 .
$$

Let $x_{0} \in \Gamma$ be a free boundary point, if $x_{0} \in \Gamma_{1} \backslash \Gamma_{2}$, or if $x_{0} \in \Gamma_{2} \backslash \Gamma_{1}$, then locally we are in the setting of the classical obstacle problem. In this case the known regularity theory for the classical obstacle problem (see 4]) can be applied to analyse the free boundary $\Gamma$ in a neighbourhood of $x_{0}$. Hence we are more curious about the behaviour of the free boundary at the points $x_{0} \in \Gamma_{1} \cap \Gamma_{2}=\partial \Lambda$. In this article we focus on the case when $x_{0} \in \Gamma_{1} \cap \Gamma_{2}$ is an isolated point of $\Lambda$. The work is inspired by the following example of a homogeneous of degree two solution in $\mathbb{R}^{2}$,

$$
u_{0}(x)=x_{1}^{2} \operatorname{sgn}\left(x_{1}\right)+x_{2}^{2} \operatorname{sgn}\left(x_{2}\right),
$$

where the obstacles $p^{1}(x)=-p^{2}(x)=-x_{1}^{2}-x_{2}^{2}$, and $\Lambda=\{0\}$. Example (1.6) has also been considered in [1, when investigating the optimal regularity in the optimal switching problem. The optimal switching problem and the double obstacle problem are related, and we see that in both cases the solution shows a new type of behaviour at isolated points of $\Lambda$. The function $u_{0}$ is a motivational example for double-cone solutions, see Definition 3.5 .

Before proceeding to the results obtained in the paper, let us mention that in the recent paper [6] the regularity of the free boundary for the double obstacle problem is studied by relaxing one of the obstacles. Under a thickness assumption, the authors in [6] show that the possible blow-ups are halfspace solutions, and prove the $C^{1}$-regularity of the free boundary. 


\subsection{Summary of the results}

We consider a normalised double obstacle problem in dimension $n=2$, with polynomial obstacles $p^{1} \leq p^{2}$

$$
\Delta u=\lambda_{1} \chi_{\left\{u=p^{1}\right\}}+\lambda_{2} \chi_{\left\{u=p^{2}\right\}},
$$

where $\lambda_{1}=\Delta p^{1}<0$ and $\lambda_{2}=\Delta p^{2}>0$ are constants. Furthermore, we assume that $p^{1}$ and $p^{2}$ meet at a single point, i.e. $p^{1}(x)=p^{2}(x)$ iff $x=x_{0}$.

Without loss of generality, we may assume that $x_{0}=0$, and the polynomials $p^{i}$ are of the form

$$
p^{1}(x)=a_{1} x_{1}^{2}+c_{1} x_{2}^{2} \text { and } p^{2}(x)=a_{2} x_{1}^{2}+c_{2} x_{2}^{2},
$$

where

$$
a_{1}+c_{1}<0, a_{2}+c_{2}>0 \text {, and } a_{1}<a_{2}, c_{1}<c_{2} .
$$

The paper is structured as follows. In Section 2 we study the normalised double obstacle problem (1.7). We show that the blow-ups of the solution to the normalised double obstacle are homogeneous of degree two functions via Weiss' monotonicity formula.

Knowing that the blow-up solutions are homogeneous of degree two functions, in Section 3 we make a complete characterisation of possible blow-ups in dimension $n=2$ (Theorem 3.8 and Theorem 3.11). In particular we see that there exist homogeneous of degree two global solutions of a new type. We call these solutions double-cone solutions, since the coincidence sets $\left\{u=p^{1}\right\}$ and $\left\{u=p^{2}\right\}$ are cones with a common vertex at the origin. We show that there exist double-cone solutions if and only if the following polynomial

$$
P=P\left(x_{1}, x_{2}\right) \equiv p^{1}\left(x_{1}, x_{2}\right)+p^{2}\left(x_{2}, x_{1}\right)=\left(a_{1}+c_{2}\right) x_{1}^{2}+\left(a_{2}+c_{1}\right) x_{2}^{2}
$$

has no sign (Corollary [3.9). The existence of halfspace solutions corresponding to $p^{i}$ is also studied (Theorem 3.11 and Corollary 3.12). In particular, we see that a halfspace solution to the obstacle problem with $p^{1}$ (or $-p^{2}$ ) is not necessarily a halfspace solution to the double obstacle problem with obstacles $p^{1} \leq p^{2}$.

Given obstacles (1.8), there are three different cases, depending on the coefficients of $p^{i}$, that describe the possible blow-up solutions for the double obstacle problem with $p^{1} \leq p^{2}$. We show the uniqueness of blow-up limits and analyse the behaviour of the free boundary in these three cases separately.

Case 1: If $P \equiv 0$, there are infinitely many double-cone solutions. This is perhaps the most interesting case, it is studied in Section 4 . This case can be reduced to the double obstacle problem with obstacles $p^{1}(x)=-x_{1}^{2}-x_{2}^{2}, p^{2}(x)=x_{1}^{2}+x_{2}^{2}$. By using a version of a flatness improvement argument, we show that if the solution is close to a double-cone solution in $B_{1}$, then the blow-up at the origin is unique. Furthermore, employing the known regularity theory for the free boundary in the classical problem, we derive that the free boundary $\Gamma$ for the double obstacle problem is a union of four $C^{1, \gamma}$-graphs meeting at the origin, see Theorem4.10. Neither $\Gamma_{1}$ nor $\Gamma_{2}$ is flat at the origin, and they meet at right angles, see Figure 4.1.

In this case there are infinitely many rotationally invariant halfspace solutions $u$ corresponding to $p^{1}$ (or $p^{2}$ ), and the set $\left\{u=p^{2}\right\}$ (or $\left\{u=p^{1}\right\}$ ) is a halfline. Via a flatness improvement argument, we show that if the solution to the double is close to a halfspace solution corresponding to $p^{1}$, then $\Gamma_{1}$ is a $C^{1, \gamma}$-curve in a neighbourhood of the origin. The proof of the last statement is the same in all three cases.

Case 2: If $P$ changes the sign, i. e. $D^{2} P$ has two eigenvalues with opposite sign, then there are only four double-cone solutions, and it follows that the blow-up at the origin is unique (Theorem 5.1). Furthermore, we show that if the solution to the double obstacle problem has a 
double-cone blow-up limit, then locally the free boundary consists of four $C^{1, \gamma}$-curves, meeting at the origin.

In Case 2 there are infinitely many halfspace solutions corresponding to $p^{i}$, which are not rotationally invariant on the plane, i.e. the rotation of $\Gamma_{i}$ can be performed only inside a fixed cone. Hence not every direction on the plane gives a halfspace solution.

Case 3: The polynomial $P$ has a sign. There are no double-cone solutions in this case. We show that if $P \geq 0$, then there are infinitely many rotationally invariant halfspace solutions corresponding to $p^{1}$. Furthermore, if $D^{2} P$ is a positive definite matrix (both eigenvalues are positive) then there are no halfspace solutions corresponding to the upper obstacle $p^{2}$. Similarly, if $P(x) \leq 0$ there are infinitely many halfspace solutions corresponding to $p^{2}$, and if $D^{2} P<0$, there are no halfspace solutions corresponding to the lower obstacle $p^{1}$. Hence the solution chooses the obstacle having lower curvature.

Let us also mention an important property of the double obstacle problem, following from our discussion of Cases 1, 2 and 3. Let $\varepsilon$ be an arbitrary number, $|\varepsilon|<<1$. Then for polynomials $p^{1}(x)=-x_{1}^{2}-x_{2}^{2}, p^{2}(x)=x_{1}^{2}+x_{1}^{2}$ there exist infinitely many double-cone solutions. While when we look at the double obstacle problem with $p^{1}=-x_{1}^{2}-x_{2}^{2}$ and $\tilde{p}^{2}=(1-\varepsilon) x_{1}^{2}+(1+\varepsilon) x_{2}^{2}$ there are only four double-cone solutions, and for $p^{1}=-x_{1}^{2}-x_{2}^{2}$ and $\bar{p}^{2}=(1+\varepsilon) x_{1}^{2}+(1+\varepsilon) x_{2}^{2}$ there are none. This property of the double obstacle problem is quite surprising and unexpected. It reveals the instability of the solutions in the sense that changing the obstacles slightly, may change the solution and the free boundary significantly.

It is an interesting question to investigate double-cone solutions also in higher dimensions. In the end of the paper we give an example of a three-dimensional double-cone solution. The complete analysis of blow-up solutions for the double obstacle problem in $\mathbb{R}^{3}$ we leave for a future publication.

\section{Acknowledgements}

The paper is a part of my doctoral thesis, written at KTH, Royal Institute of Technology in Stockholm. I am grateful to my advisor, Prof. Dr. John Andersson, for his guidance and support throughout the project.

I would also like to thank Dr. Erik Lindgren and Prof. Dr. Henrik Shahgholian for reading a preliminary version of the manuscript and for their valuable feedback.

\section{Weiss' energy functional for the double obstacle problem}

In this section we study the behaviour of the solutions locally at free boundary points via Weiss' monotonicity formula.

Let $u$ be a solution to the double problem in $\Omega$, with obstacles

$$
\psi^{1} \leq \psi^{2}, \psi^{1}, \psi^{2} \in C^{2}(\Omega), \Lambda=\left\{\psi^{1}=\psi^{2}\right\} \neq \emptyset .
$$

Fix any $x_{0} \in \Gamma \cap \partial \Lambda$ and assume that $B_{1}\left(x_{0}\right) \subset \Omega$. Denote by

$$
u_{r, x_{0}}:=\frac{u\left(r x+x_{0}\right)-u\left(x_{0}\right)-r \nabla u\left(x_{0}\right) \cdot x}{r^{2}}, \text { for all } 0<r<1, \text { and } x_{0} \in \Gamma .
$$

Without loss of generality, assume that $x_{0}=0$ and $B_{1} \subset \Omega$. Furthermore, by subtracting a first order polynomial from $u$, we may assume that $u(0)=|\nabla u(0)|=0$. Recalling that $u \in C_{l o c}^{1,1}$, we 
obtain $\psi^{1}(0)=\psi^{2}(0)=u(0)=0$ and $\left|\nabla \psi^{1}(0)\right|=\left|\nabla \psi^{2}(0)\right|=|\nabla u(0)|=0$. Denote by

$$
u_{r}(x):=u_{r, 0}=\frac{u(r x)}{r^{2}} .
$$

It follows from equation (1.2) and assumption (2.1), that $\lambda_{1}=\Delta \psi^{1}(0) \leq 0$ and $\lambda_{2}=$ $\Delta \psi^{2}(0) \geq 0$. In particular, if $0 \in \partial \Lambda^{\circ}$, then $\lambda_{1}=\lambda_{2}=0$.

Lemma 2.1. Consider the following normalised double obstacle problem

$$
\Delta u=\lambda_{1} \chi_{\left\{u=\psi^{1}\right\}}+\lambda_{2} \chi_{\left\{u=\psi^{2}\right\}}, \text { in } B_{1}
$$

where $\psi^{i} \in C^{2}\left(B_{1}\right)$, and assume that

$$
\lambda_{1}:=\Delta \psi^{1} \leq 0 \text { and } \lambda_{2}:=\Delta \psi^{2} \geq 0 \text { are constants. }
$$

Define Weiss' energy functional for the function $u$ and $0<r \leq 1$ at the origin as follows

$$
\begin{aligned}
W(u, r, 0) & :=\frac{1}{r^{n+2}} \int_{B_{r}}|\nabla u|^{2} d x-\frac{2}{r^{n+3}} \int_{\partial B_{r}} u^{2} d \mathcal{H}^{n-1} \\
& +\frac{1}{r^{n+2}} \int_{B_{r}} 2 \lambda_{1} u \chi_{\left\{u=\psi^{1}\right\}}+2 \lambda_{2} u \chi_{\left\{u=\psi^{2}\right\}} d x .
\end{aligned}
$$

Then

$$
\frac{d}{d r} W(u, r, 0)=2 r \int_{\partial B_{1}}\left(\frac{d u_{r}}{d r}\right)^{2} d \mathcal{H}^{n-1} \geq 0 .
$$

Proof. After a change of variable in (2.5) we obtain the following scaling property for Weiss' energy functional

$$
\begin{array}{r}
W(u, r, 0)=W\left(u_{r}, 1,0\right)=\int_{B_{1}}\left|\nabla u_{r}\right|^{2} d x-2 \int_{\partial B_{1}} u_{r}^{2} d \mathcal{H}^{n-1} \\
+\int_{B_{1}} 2 \lambda_{1} u_{r} \chi_{\left\{u_{r}=\psi_{r}^{1}\right\}}+2 \lambda_{2} u_{r} \chi_{\left\{u_{r}=\psi_{r}^{2}\right\}} d x .
\end{array}
$$

Hence

$$
\begin{array}{r}
\frac{d}{d r} W(u, r, 0)=\frac{d}{d r} W\left(u_{r}, 1,0\right)=\int_{B_{1}} \frac{d}{d r}\left|\nabla u_{r}\right|^{2} d x-2 \int_{\partial B_{1}} \frac{d u_{r}^{2}}{d r} d \mathcal{H}^{n-1} \\
\quad+2 \int_{B_{1}}\left(\lambda_{1} \chi_{\left\{u_{r}=\psi_{r}^{1}\right\}}+\lambda_{2} \chi_{\left\{u_{r}=\psi_{r}^{2}\right\}}\right) \frac{d u_{r}}{d r} d x=2 \int_{B_{1}} \nabla \frac{d u_{r}}{d r} \nabla u_{r} d x \\
-4 \int_{\partial B_{1}} \frac{d u_{r}}{d r} u_{r} d \mathcal{H}^{n-1}+2 \int_{B_{1}}\left(\lambda_{1} \chi_{\left\{u_{r}=\psi_{r}^{1}\right\}}+\lambda_{2} \chi_{\left\{u_{r}=\psi_{r}^{2}\right\}}\right) \frac{d u_{r}}{d r} d x
\end{array}
$$

By Green's formula

$$
\int_{B_{1}} \nabla u_{r} \nabla \frac{d u_{r}}{d r} d x=-\int_{B_{1}} \frac{d u_{r}}{d r} \Delta u_{r} d x+\int_{\partial B_{1}} \frac{d u_{r}}{d r} \frac{\partial u_{r}}{\partial \nu} d \mathcal{H}^{n-1} .
$$

Therefore

$$
\begin{gathered}
\frac{d}{d r} W(u, r, 0)=2 \int_{\partial B_{1}} \frac{d u_{r}}{d r}\left(\frac{\partial u_{r}}{\partial \nu}-2 u_{r}\right) d \mathcal{H}^{n-1} \\
+2 \int_{B_{1}} \frac{d u_{r}}{d r}\left(-\Delta u_{r}+\lambda_{1} \chi_{\left\{u_{r}=\psi_{r}^{1}\right\}}+\lambda_{2} \chi_{\left\{u_{r}=\psi_{r}^{2}\right\}}\right) d x .
\end{gathered}
$$


Since $u$ solves (2.3), equation 2.8 can be abbreviated to

$$
\frac{d}{d r} W(u, r, 0)=2 \int_{\partial B_{1}} \frac{d u_{r}}{d r}\left(\frac{\partial u_{r}}{\partial \nu}-2 u_{r}\right) d \mathcal{H}^{n-1} .
$$

Let us observe that

$$
\begin{array}{r}
\int_{\partial B_{1}} \frac{d u_{r}}{d r}\left(\frac{\partial u_{r}}{\partial \nu}-2 u_{r}\right) d \mathcal{H}^{n-1}=\int_{\partial B_{1}} \frac{d u_{r}}{d r}\left(x \cdot \nabla u_{r}-2 u_{r}\right) d \mathcal{H}^{n-1} \\
=\int_{\partial B_{1}} r\left(\frac{d u_{r}}{d r}\right)^{2} d \mathcal{H}^{n-1} .
\end{array}
$$

Equations (2.10) and (2.9) together imply the desired identity, (2.6).

\section{Characterisation of blow-ups in $\mathbb{R}^{2}$}

Given second degree polynomials $p^{1} \leq p^{2}$, satisfying (2.4), let $u$ be the solution to the normalised double obstacle problem (2.3) with $p^{1}, p^{2}$. Let $0 \in \Gamma_{1} \cap \Gamma_{2}$ be a free boundary point. By subtracting a first order polynomial from $p^{1}, p^{2}$ and $u$, and recalling that $u \in C^{1,1}$, we may assume

$$
u(0)=p^{1}(0)=p^{2}(0)=0 \text { and }|\nabla u(0)|=\left|\nabla p^{1}(0)\right|=\left|\nabla p^{2}(0)\right|=0 .
$$

Hence $p^{1}$ and $p^{2}$ are homogeneous second degree polynomials.

It follows from Lemma 2.1 that $W(u, r, 0)$ is a nondecreasing absolutely continuous function in the interval $(0,1)$. Hence there exists

$$
\lim _{r \rightarrow 0} W(u, r, 0):=W(u, 0+, 0) .
$$

Since $u \in C_{l o c}^{1,1}$, we may conclude that $\left\|u_{r}\right\|_{C^{1,1}}$ is uniformly bounded for small $r>0$. Therefore through a subsequence $u_{r}$ converges in $C^{1, \alpha}\left(B_{1}\right)$. Let $u_{0}$ be a blow-up of $u$ at the origin;

$$
\frac{u\left(r_{j} x\right)}{r_{j}^{2}} \rightarrow u_{0} \text { in } C^{1, \alpha}\left(B_{1}\right),
$$

for a sequence $r_{j} \rightarrow 0+$, as $j \rightarrow \infty$. Then (3.3) implies that for any fixed $0<r<1$

$$
W\left(u_{0}, r, 0\right)=\lim _{j \rightarrow \infty} W\left(u_{r_{j}}, r, 0\right) \stackrel{\sqrt{2.7}}{=} \lim _{j \rightarrow \infty} W\left(u, r r_{j}, 0\right) \stackrel{\sqrt{3.2)}}{=} W(u, 0+, 0) .
$$

Thus $W\left(u_{0}, r, 0\right)$ has a constant value for all $0<r<1$, and $\frac{d}{d r} W\left(u_{0}, r, 0\right)=0$. Note that $u_{0}$ is a global solution, i.e. solution in $\mathbb{R}^{n}$ to the double obstacle problem with the same obstacles, $p^{1}$ and $p^{2}$. Applying Lemma 2.1 for the solution $u_{0}$, we may conclude from (2.6), that

$$
\frac{d}{d r}\left(\frac{u_{0}(r x)}{r^{2}}\right)=0, \text { for any } r>0 .
$$

Hence $u_{0}$ is a homogeneous of degree two function, which means that

$$
u_{0}(x)=\frac{u_{0}(r x)}{r^{2}}, \text { for any } x \in \mathbb{R}^{n} \text { and } r>0 .
$$

It follows that $\Delta u_{0}(r x)=\Delta u_{0}(x)$, for any $x \in \mathbb{R}^{n}$ and $r>0$. In other words $\Delta u_{0}$ is identically constant on the lines passing through the origin, and therefore the free boundary of $u_{0}$ is lying on straight lines passing through the origin. 


\subsection{Examples}

In this section we study motivational examples of homogeneous of degree two global solutions in $\mathbb{R}^{2}$, assuming that $\Lambda=\{0\}$.

It is well known that the (single) obstacle problem has two types of blow-ups; polynomial and halfspace solutions. The first obvious question is the following; if or when the halfspace solutions to the obstacle problem are also solutions to the double obstacle problem, and second, if the double obstacle problem has any other type of blow-ups which the obstacle problem does not.

By using comparison principles, it is easy to see that if $u$ is a polynomial solution to the double obstacle problem (2.3), then $u \equiv p^{1}, u \equiv p^{2}$ or otherwise $u$ is a homogeneous of degree two harmonic polynomial in $\mathbb{R}^{2}$, such that $p^{1} \leq u \leq p^{2}$.

Let us also recall the definition of a halfspace solution in $\mathbb{R}^{n}$ (or halfplane in $\operatorname{dim} n=2$ ).

Definition 3.1. Let $p^{1} \leq p^{2}$ be given homogeneous degree two polynomials in $\mathbb{R}^{n}$, satisfying $\lambda_{1}=\Delta p^{1}<0$ and $\lambda_{2}=\Delta p^{2}>0$. We say that $u$ is a halfspace solution to the double obstacle problem corresponding to the lower obstacle $p^{1}$, if $u \leq p^{2}$ in $\mathbb{R}^{n}$, and $u-p^{1}=-\frac{\lambda_{1}}{2}(x \cdot e)_{+}^{2}$, where $e$ is a unit vector in $\mathbb{R}^{n}$. Similarly, $u$ is a halfspace solution corresponding to the upper obstacle $p^{2}$, if $u \geq p^{1}$ in $\mathbb{R}^{n}$, and $p^{2}-u=\frac{\lambda_{2}}{2}(x \cdot e)_{+}^{2}$.

It follows from Definition 3.1 that if $u$ is a halfspace solution corresponding to $p^{1}$, then $\Delta u=\lambda_{1} \chi_{\{(x \cdot e)<0\}}$, and $u<p^{2}$ a.e.. Similarly, if $u$ is a halfspace solution corresponding to $p^{2}$, then $\Delta u=\lambda_{2} \chi_{\{(x \cdot e)<0\}}$, and $u>p^{1}$ a.e..

In the following examples instead of our usual notation $x=\left(x_{1}, x_{2}\right) \in \mathbb{R}^{2}$, the pair $(x, y)$ represents a point in $\mathbb{R}^{2}$. It is done to make the pictures clearer, and we hope it will not be confusing later on.

Example 3.2. Let us study some explicit homogeneous degree two solutions to the double obstacle problem in $\mathbb{R}^{2}$, with fixed obstacles $p^{1}(x, y)=-x^{2}-y^{2}, p^{2}(x, y)=x^{2}+y^{2}$.

Observe that $u_{0}=-x^{2}+\operatorname{sgn}(y) y^{2}$ and $u_{0}=\operatorname{sgn}(x) x^{2}+y^{2}$ are halfspace solutions corresponding to $p^{1}=-x^{2}-y^{2}$ and to $p^{2}=x^{2}+y^{2}$ respectively;

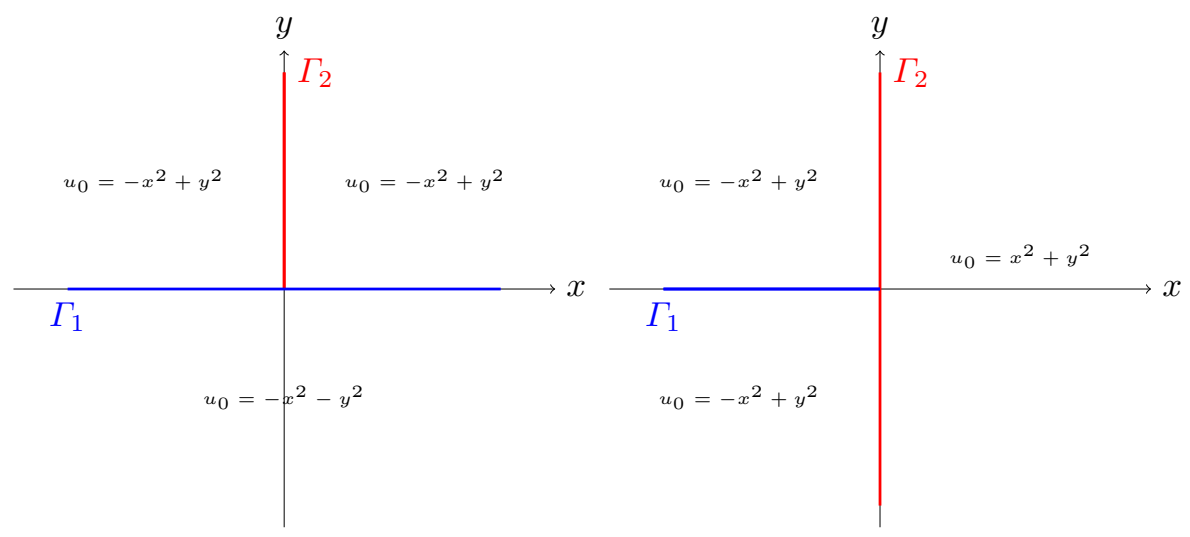

Figure 3.1: Examples of halfspace solutions.

Now let us look at the following two explicit solutions, which obviously are not halfspace solutions. 

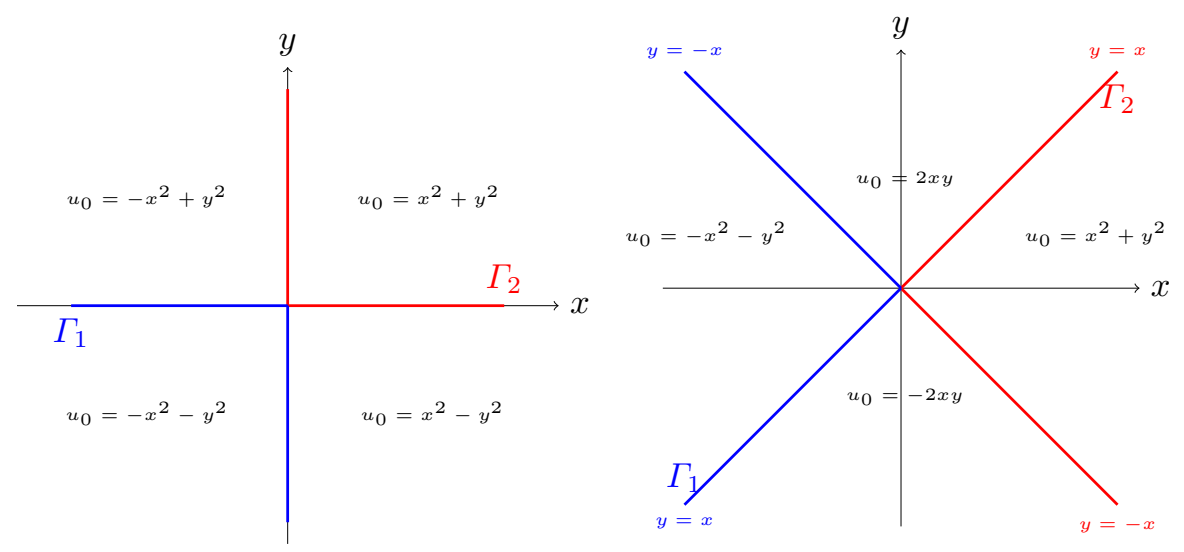

Figure 3.2: New, interesting type of solutions.

We see that $\Gamma=\Gamma_{1} \cup \Gamma_{2}$ consists of two lines meeting at right angles, and $\Gamma_{1} \cap \Gamma_{2}=\{0\}=\Lambda$. Actually there are many more solutions, for example consider the following global solutions
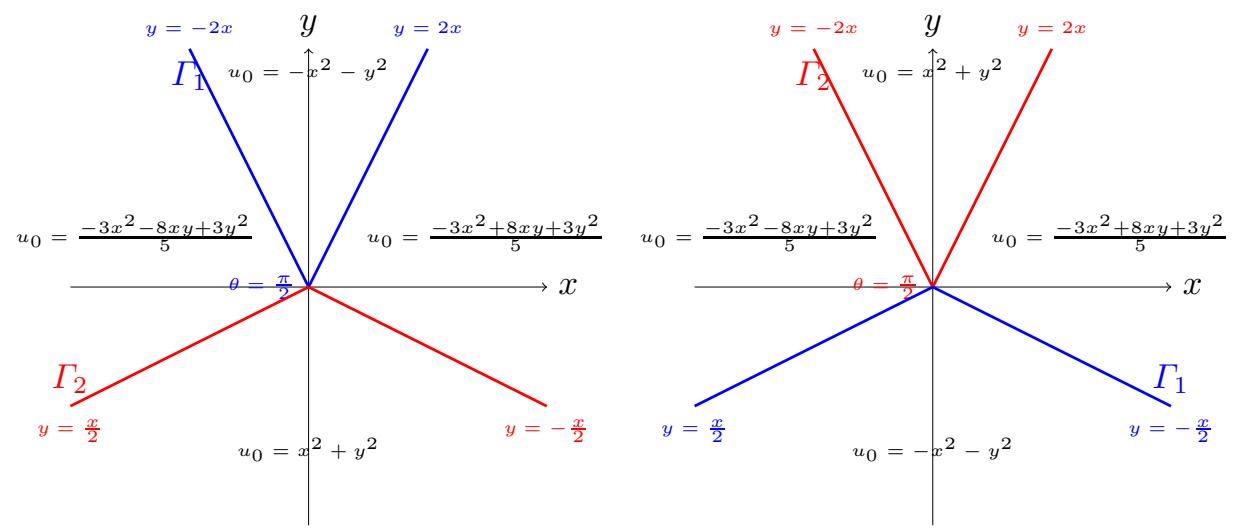

Figure 3.3: In this example we see that the cone $\left\{u_{0}=p^{1}\right\}\left(\left\{u_{0}=p^{2}\right\}\right)$ does not have a fixed opening angle. Actually the opening angle can take any value in the closed interval $[0, \pi]$.

We see that in all the examples discussed above there is one common property: in the halfplane $x \geq 0$ the lines $\Gamma_{1}$ and $\Gamma_{2}$ intersect at a right angle, later on we will provide a rigorous argument for this.

Let us study two more examples, where the free boundary shows a different behaviour.

Example 3.3. Let $p^{1}(x, y)=-x^{2}-y^{2}$ and $p^{2}(x, y)=2 x^{2}+2 y^{2}$. Assume that $u^{0}$ is a homogeneous of degree two solution to the double obstacle problem with obstacles $p^{1}$ and $p^{2}$ in $\mathbb{R}^{2}$, then $\Gamma_{2}=\{0\}$. In this case if a blow-up is not a polynomial, then it is a halfspace solution corresponding to $p^{1}$.

It is easy to verify that there is no second order harmonic polynomial in $\mathbb{R}^{2}$, satisfying $p^{1} \leq q \leq p^{2}$ and such that the polynomials $p^{2}-q$ and $q-p^{1}$ both have roots of multiplicity two. 
Furthermore, in this case there are no halfspace solutions corresponding to $p^{2}$.
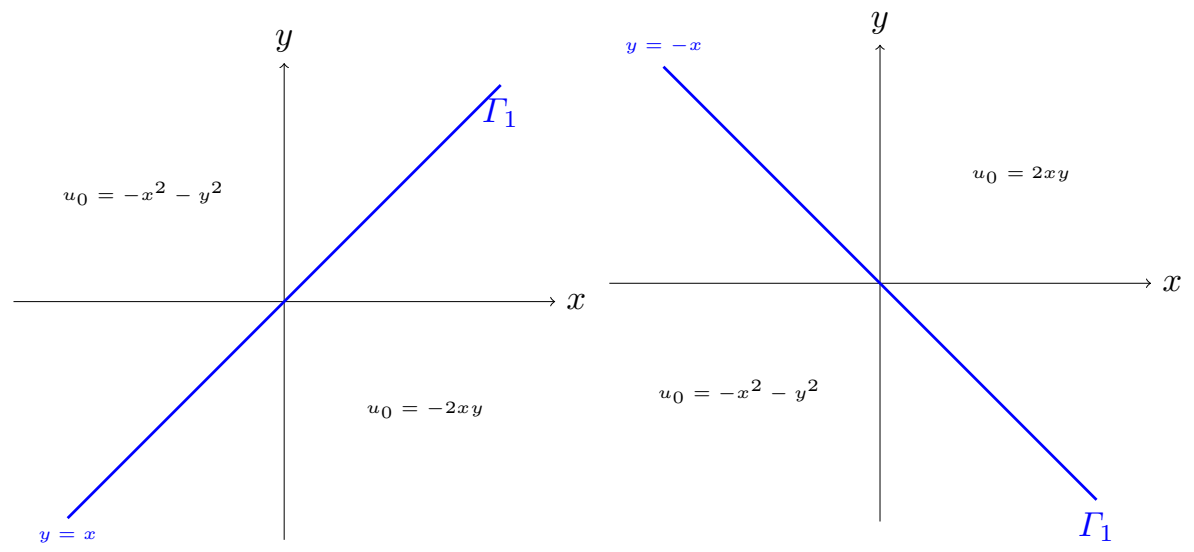

Figure 3.4: Examples of halfspace solutions.

Example 3.4. The following functions are homogeneous global solutions to the double obstacle problem with $p^{1}(x, y)=-x^{2}-y^{2}$ and $p^{2}(x, y)=2 x^{2}$.
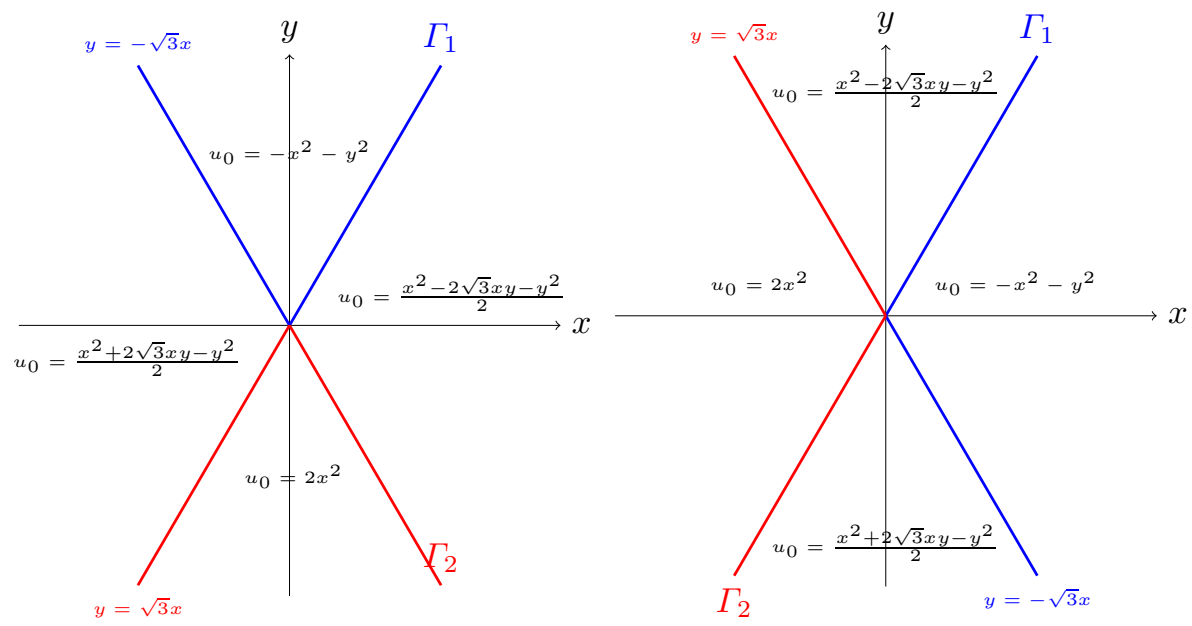

Figure 3.5: The noncoincidence set is a cone with an opening angle $2 \pi / 3$ or $\pi / 3$.

\subsection{Double-cone solutions}

Let $p^{1} \leq p^{2}$ be given polynomials,

$$
p^{i}(x) \equiv a_{i} x_{1}^{2}+2 b_{i} x_{1} x_{2}+c_{i} x_{2}^{2}, \text { for } i=1,2,
$$

Consider the following normalised double obstacle problem in $\mathbb{R}^{2}$ with obstacles $p^{1}, p^{2}$;

$$
p^{1} \leq u \leq p^{2}, \Delta u=\lambda_{1} \chi_{\left\{u=p^{1}\right\}}+\lambda_{2} \chi_{\left\{u=p^{2}\right\}},
$$

where

$$
\lambda_{1}:=\Delta p^{1}=2\left(a_{1}+c_{1}\right)<0 \text { and } \lambda_{2}:=\Delta p^{2}=2\left(a_{2}+c_{2}\right)>0 .
$$


We saw in Example 3.2 and Example 3.4 that for the double obstacle problem there exist global solutions for which the coincidence sets $\left\{u=p^{1}\right\}$ and $\left\{u=p^{2}\right\}$ are halfcones with a common vertex at the origin.

Definition 3.5. Let $u$ be a global solution to the normalised double obstacle problem with obstacles $p^{1} \leq p^{2}$. We say that $u$ is a double-cone solution, if both $\left\{u=p^{1}\right\}$ and $\left\{u=p^{2}\right\}$ are halfcones with a common vertex.

Remark 3.6. Definition 3.5 is applicable also in higher dimensions. In the last section we will show the existence of three-dimensional double-cone solutions.

In this section our aim is to describe the possible blow-ups for a solution to the double obstacle problem in $\mathbb{R}^{2}$. In particular, we are interested to study the case when the double-cone solutions do exist. It is easy to verify that if $\lambda_{1}=0$ or $\lambda_{2}=0$, there are no double-cone solutions, explaining our assumption (3.6).

A simple calculation shows that if $p^{1}=p^{2}$ on a line, then there are no double-cone solutions. Hence we assume that $p^{1}$ and $p^{2}$ meet only at the origin, in other words the matrix $D^{2}\left(p^{2}-p^{1}\right)$ is positive definite.

Without loss of generality we may assume that $b_{1}=b_{2}=b$ in (3.4). Otherwise, if $b_{2}-b_{1} \neq 0$, we can rotate the coordinate system with an angle $\theta, \frac{\cos 2 \theta}{\sin 2 \theta}=\frac{a_{2}-a_{1}-c_{2}+c_{1}}{2\left(b_{1}-b_{2}\right)}$, and obtain $b_{1}=b_{2}$ in the new system. Furthermore, we may subtract a harmonic polynomial $h(x)=2 b x_{1} x_{2}$ from $p^{1}$, $p^{2}$ and $u$, then consider instead the polynomials $p^{1}-h$ and $p^{2}-h$, thus obtaining $b=0$. Instead of $u$, we are studying the solution $u-h$, but still call it $u$.

We saw that it is enough to study the blow-up solutions of the double obstacle problem with obstacles having the form

$$
p^{1}(x)=a_{1} x_{1}^{2}+c_{1} x_{2}^{2} \quad \text { and } \quad p^{2}(x)=a_{2} x_{1}^{2}+c_{2} x_{2}^{2} .
$$

According to our assumption, the matrix $A:=D^{2}\left(p^{2}-p^{1}\right)$ is positive definite, hence

$$
a_{2}>a_{1}, \quad c_{2}>c_{1} .
$$

and by (3.6),

$$
a_{1}+c_{1}<0, \text { and } a_{2}+c_{2}>0 .
$$

If $u$ is a double-cone solution in $\mathbb{R}^{2}$, then the noncoincidence set $\Omega_{12}=\left\{p^{1}<u<p^{2}\right\}$ consists of two halfcones $\mathcal{S}_{1}$ and $\mathcal{S}_{2}$, having a common vertex. So the expression "double-cone" may refer to the cones $\mathcal{S}_{i}$ as well. The following lemma is the main step to the investigation of double-cone solutions in $\mathbb{R}^{2}$.

Lemma 3.7. Let $p^{1}(x)=a_{1} x_{1}^{2}+c_{1} x_{2}^{2}$ and $p^{2}(x)=a_{2} x_{1}^{2}+c_{2} x_{2}^{2}$ be given polynomials, satisfying (3.8) and (3.9). Assume that there exists a pair $(q, \mathcal{S})$, where $\mathcal{S}$ is an open sector in $\mathbb{R}^{2}$, with the edges lying on the lines $x_{2}=m x_{1}$ and $x_{2}=k x_{1}$, and $q$ is a harmonic homogeneous of degree two function in $\mathcal{S}$. Moreover, assume that

$$
p^{1} \leq q \leq p^{2} \text { in } \mathcal{S}
$$

and the following boundary conditions hold;

$$
q-p^{1}=0, \nabla\left(q-p^{1}\right)=0 \text { on } x_{2}=m x_{1},
$$

and

$$
q-p^{2}=0, \nabla\left(q-p^{2}\right)=0 \text { on } x_{2}=k x_{1} .
$$


Then $q=\alpha x_{1}^{2}+2 \beta x_{1} x_{2}-\alpha x_{2}^{2}$, where $\alpha$ and $\beta$ are real numbers solving

$$
\begin{gathered}
\beta^{2}=-\left(\alpha-a_{1}\right)\left(\alpha+c_{1}\right)=-\left(\alpha-a_{2}\right)\left(\alpha+c_{2}\right) \geq 0, \\
\max \left(a_{1},-c_{2}\right) \leq \alpha \leq \min \left(a_{2},-c_{1}\right),
\end{gathered}
$$

and

$$
\alpha\left(c_{1}-a_{1}-c_{2}+a_{2}\right)=a_{1} c_{1}-a_{2} c_{2} .
$$

The numbers $m$ and $k$ are given by

$$
m=\frac{\beta}{\alpha+c_{1}} \text { and } k=\frac{\beta}{c_{2}+\alpha} .
$$

Furthermore, the coefficients of $p^{1}$ and $p^{2}$ satisfy the following inequality

$$
\left(a_{1}+c_{2}\right)\left(c_{1}+a_{2}\right) \leq 0 .
$$

Proof. Let us note that harmonic homogeneous of degree two functions in a sector are second degree polynomials of the form $q(x)=\alpha x_{1}^{2}+2 \beta x_{1} x_{2}-\alpha x_{2}^{2}$, where $\alpha$ and $\beta$ are real numbers. By assumption (3.10),

$$
\begin{gathered}
q-p^{1}=\left(\alpha-a_{1}\right) x_{1}^{2}+2 \beta x_{1} x_{2}-\left(\alpha+c_{1}\right) x_{2}^{2} \geq 0, \text { and } \\
p^{2}-q=\left(a_{2}-\alpha\right) x_{1}^{2}-2 \beta x_{1} x_{2}+\left(c_{2}+\alpha\right) x_{2}^{2} \geq 0 \text { in } \mathcal{S} .
\end{gathered}
$$

Denote by $t=\frac{x_{2}}{x_{1}}$, and observe that (3.11) implies that the following quadratic polynomial

$$
\frac{q-p^{1}}{x_{1}^{2}}=-\left(\alpha+c_{1}\right) t^{2}+2 \beta t+\alpha-a_{1}
$$

has a multiple root at the point $t=m$. By an elementary calculation we obtain

$$
\beta^{2}=-\left(\alpha-a_{1}\right)\left(\alpha+c_{1}\right), \text { and } \frac{q-p^{1}}{x_{1}^{2}}=-\left(\alpha+c_{1}\right)(t-m)^{2} .
$$

Hence the inequality $q-p^{1} \geq 0$ in $\mathcal{S}$ implies $q-p^{1} \geq 0$ in $\mathbb{R}^{2}$. Therefore we may conclude that

$$
-\alpha-c_{1} \geq 0, \alpha-a_{1} \geq 0, \beta^{2}=-\left(\alpha-a_{1}\right)\left(\alpha+c_{1}\right), \text { and } m=\frac{\beta}{\alpha+c_{1}} .
$$

Similarly, (3.12) implies that the following quadratic polynomial

$$
\frac{p^{2}-q}{x_{1}^{2}}=\left(c_{2}+\alpha\right) t^{2}+-2 \beta t+a_{2}-\alpha
$$

has a multiple root at the point $t=k$. Hence $\beta^{2}=\left(a_{2}-\alpha\right)\left(c_{2}+\alpha\right)$, and the inequality $p^{2}-q \geq 0$ in $\mathcal{S}$ implies $p^{2}-q \geq 0$ in $\mathbb{R}^{2}$. Therefore, by a similar argument as the one leading to (3.18), we get

$$
c_{2}+\alpha \geq 0, a_{2}-\alpha \geq 0, \beta^{2}=\left(a_{2}-\alpha\right)\left(c_{2}+\alpha\right), \text { and } k=\frac{\beta}{c_{2}+\alpha} .
$$

Let us also observe that if $\alpha=-c_{1}$, then $p^{1}(x)=q(x)$ implies $x_{1}=0$, similarly, if $\alpha=-c_{2}$, then $p^{2}(x)=q(x)$ implies $x_{1}=0$. Hence (3.16) makes sense even if $\alpha=-c_{1}$ or $\alpha=-c_{2}$. 
Assuming that there exists $(q, \mathcal{S})$ satisfying (3.10), (3.11), (3.12), we derived (3.18) and (3.19), which in particular imply (3.13), (3.14) and (3.16). It follows from (3.13), that

$$
\alpha^{2}-a_{1} \alpha+c_{1} \alpha-a_{1} c_{1}=\alpha^{2}-a_{2} \alpha+c_{2} \alpha-a_{2} c_{2},
$$

hence $\alpha$ solves equation (3.15). As we see equation (3.15) is contained in (3.13), we stated (3.15) only for the future references.

It remains to prove the inequality (3.17), which is a necessary condition for the existence of $\alpha, \beta$, thus for $(q, \mathcal{S})$. We discuss two cases. $i)$ If

$$
c_{1}-a_{1}-c_{2}+a_{2}=0,
$$

it follows from equation (3.15) that

$$
a_{2} c_{2}=a_{1} c_{1} .
$$

If $a_{1}=0$, then $a_{2}<0$ by (3.8), therefore $c_{2}=0$, and (3.17) holds. Otherwise, if $a_{1} \neq 0$, let $a_{2}=l a_{1}$, then $l \neq 1$ by (3.8). Hence $c_{1}=l c_{2}$ according to (3.21). Now (3.20) implies that $(l-1)\left(a_{1}+c_{2}\right)=0$, since $l \neq 1$, we obtain $a_{2}+c_{1}=0$, and (3.17) holds.

ii) If $c_{1}-a_{1}-c_{2}+a_{2} \neq 0$, then equation (3.15) implies that

$$
\alpha=\frac{a_{2} c_{2}-a_{1} c_{1}}{c_{2}+a_{1}-a_{2}-c_{1}} .
$$

By a direct computation we see that

$$
\alpha-a_{1}=\frac{\left(a_{2}-a_{1}\right)\left(a_{1}+c_{2}\right)}{c_{2}+a_{1}-a_{2}-c_{1}}, \text { and } \alpha+c_{1}=\frac{\left(c_{2}-c_{1}\right)\left(a_{2}+c_{1}\right)}{c_{2}+a_{1}-a_{2}-c_{1}},
$$

by (3.13)

$$
\beta^{2}=-\frac{\left(a_{2}-a_{1}\right)\left(c_{2}-c_{1}\right)\left(a_{1}+c_{2}\right)\left(a_{2}+c_{1}\right)}{\left(c_{2}+a_{1}-a_{2}-c_{1}\right)^{2}} \geq 0 .
$$

Taking into account (3.8), we obtain the desired inequality, (3.17).

Let us observe that if $u_{0}$ is a double-cone solution (Definition [3.5), then there exist $\left(q_{1}, \mathcal{S}_{1}\right)$ and $\left(q_{2}, \mathcal{S}_{2}\right)$ as in Lemma 3.7, such that $\mathcal{S}_{1} \cap \mathcal{S}_{2}=\emptyset$, and

$$
u_{0}=q_{1} \text { in } \mathcal{S}_{1} \text { and } u_{0}=q_{2} \text { in } \mathcal{S}_{2} .
$$

According to Lemma 3.7, the inequality (3.17) is a necessary condition for the existence of double-cone solutions, in the next theorem we will discuss if (3.17) is also a sufficient condition.

Theorem 3.8. (The existence of double-cone solutions)

Let $u_{0}$ be a homogeneous of degree two global solution to the double obstacle problem with obstacles

$$
p^{1}(x)=a_{1} x_{1}^{2}+c_{1} x_{2}^{2} \text { and } p^{2}(x)=a_{2} x_{1}^{2}+c_{2} x_{2}^{2},
$$

satisfying (3.9) and (3.8). If $u_{0}$ is neither a polynomial nor a halfspace solution, then it is a double-cone solution.

Case 1) If $a_{2}+c_{1}=c_{2}+a_{1}=0$, then there are infinitely many double-cone solutions. Each of the cones $\mathcal{S}_{1}$ and $\mathcal{S}_{2}$ in (3.23) has an opening angle $\vartheta=\pi / 2$. 
Case 2) If $\left(a_{1}+c_{2}\right)\left(c_{1}+a_{2}\right)<0$, then there exist four double-cone solutions. Furthermore, the opening angle of $\mathcal{S}_{i}$, denoted by $\vartheta_{i}$, satisfies

$$
\vartheta_{1}=\vartheta_{2}=\vartheta, \text { and } \cos ^{2} \vartheta=\frac{\left(a_{1}+c_{2}\right)\left(a_{2}+c_{1}\right)}{\left(a_{1}+c_{1}\right)\left(a_{2}+c_{2}\right)} \in(0,1) .
$$

Case 3) If $\left(a_{1}+c_{2}\right)\left(c_{1}+a_{2}\right) \geq 0$, and $a_{1}+c_{2} \neq 0$ or $a_{2}+c_{1} \neq 0$, then there are no double-cone solutions.

Proof. If $u_{0}$ is neither a polynomial nor a halfspace solution, then there exists a pair $(q, \mathcal{S})$, such that

$$
u_{0}=q \text { in } \mathcal{S}, u_{0}=p^{1} \text { on }\left\{x_{2}=m x_{1}\right\}, u_{0}=p^{2} \text { on }\left\{x_{2}=k x_{1}\right\}
$$

where $S$ is a sector in $\mathbb{R}^{2}$, with edges lying on the lines $\left\{x_{2}=m x_{1}\right\}$ and $\left\{x_{2}=k x_{1}\right\}$, and $q$ is a harmonic homogeneous degree two function in $\mathcal{S}$, satisfying (3.10). Moreover, since $u_{0} \in C^{1,1}$, we obtain $\nabla q=\nabla p^{1}$ on $\left\{x_{2}=m x_{1}\right\}$ and $\nabla q=\nabla p^{2}$ on $\left\{x_{2}=k x_{1}\right\}$. Hence $q$ takes boundary conditions (3.11) and (3.12) on $\partial \mathcal{S} \subset\left\{x_{2}=m x_{1}\right\} \cup\left\{x_{2}=k x_{1}\right\}$, and therefore $(q, \mathcal{S})$ satisfies the assumptions in Lemma 3.7

According to Lemma 3.7, $q=\alpha x_{1}^{2}+2 \beta x_{1} x_{2}-\alpha x_{2}^{2}$, where $\alpha$ and $\beta$ are real numbers solving (3.13) and (3.14). The numbers $m$ and $k$, describing the sector $\mathcal{S}$, are given by (3.16).

We are looking for all possible pairs $(q, \mathcal{S})$ in terms of the parameter $\alpha$. Given $\alpha$, satisfying (3.14) and (3.15), we can find $\pm \beta$ from equation (3.13). By equation (3.16) we can identify the corresponding sectors $\mathcal{S}$.

Let us split the discussion into several cases in order to study the existence of solutions to the equation (3.15) in variable $\alpha$, satisfying inequality (3.14).

Case 1) If $a_{2}+c_{1}=c_{2}+a_{1}=0$, as in Example 3.2. Then obviously equation (3.15) becomes an identity. Hence in this case $\alpha$ can be any number satisfying (3.14), that is $a_{1} \leq \alpha \leq a_{2}$. If $\alpha=a_{1}$, then $\beta=0$ in view of (3.13), and according to (3.16), $\Gamma_{1}=\left\{x_{2}=0\right\}$. In this case $\Gamma_{2}=\left\{x_{1}=0, x_{2} \geq 0\right\}$ or $\Gamma_{2}=\left\{x_{1}=0, x_{2} \leq 0\right\}$. Analogously if $\alpha=a_{2}$ then $\Gamma_{2}=\left\{x_{2}=0\right\}$ and $\Gamma_{1}=\left\{x_{1}=0, x_{2} \geq 0\right\}$ or $\Gamma_{2}=\left\{x_{1}=0, x_{2} \leq 0\right\}$. Hence we obtain halfspace solutions, see Figure 3.1 with $\alpha=-1=a_{1}$ and $\alpha=1=a_{2}$. These can still be viewed as double-cone solutions, if we allow the cone $\mathcal{S}_{2}$ to be a halfline.

Now let us fix any $a_{1}<\alpha<a_{2}$. It follows from (3.18) and (3.19) that

$$
\beta_{ \pm}= \pm \sqrt{\left(\alpha-a_{1}\right)\left(a_{2}-\alpha\right)}
$$

and

$$
m_{ \pm}=\mp \sqrt{\frac{\alpha-a_{1}}{a_{2}-\alpha}}, \quad k_{ \pm}= \pm \sqrt{\frac{a_{2}-\alpha}{\alpha-a_{1}}} .
$$

Let us note that $m^{ \pm} k^{ \pm}=-1$, and therefore the lines $x_{2}=m^{ \pm} x_{1}$ and $x_{2}=k^{ \pm} x_{1}$ are perpendicular. Thus for a fixed $a_{1}<\alpha<a_{2}$ we obtain two polynomials

$$
q_{+}:=\alpha x_{1}^{2}+2 \beta_{+} x_{1} x_{2}-\alpha x_{2}^{2} \text { and } q_{-}:=\alpha x_{1}^{2}+2 \beta_{-} x_{1} x_{2}-\alpha x_{2}^{2} .
$$

Where $q_{+}=p^{1}$ if $x_{2}=m_{+} x_{1}, q_{+}=p^{2}$ if $x_{2}=k_{+} x_{1}$, and $q_{-}=p^{1}$ if $x_{2}=m_{-} x_{1}, q_{-}=p^{2}$ if $x_{2}=k_{-} x_{1}$. Hence for a fixed $\alpha$ there are two pairs $\left(q_{+}, \mathcal{S}_{1}\right)$ and $\left(q_{-}, \mathcal{S}_{2}\right)$ forming a single double-cone solution $u_{0}$. There are four different choices of disjoint sectors $\mathcal{S}_{1}$ and $\mathcal{S}_{2}$, satisfying (3.26). Therefore we obtain four different double-cone solutions for a fixed $a_{1}<\alpha<a_{2}$. Figure 3.3 illustrates two of them for $\alpha=-\frac{3}{5}$.

In fact we obtain more double-cone solutions by "merging" two double-cone solutions corresponding to two different values of $\alpha$. Consider the following example $u_{0}=x_{1}^{2} \operatorname{sgn}\left(x_{1}\right)+x_{2}^{2} \operatorname{sgn}\left(x_{2}\right)$ 
(see the left picture in Figure 3.2). Then $q_{1}=-x_{1}^{2}+x_{2}^{2}$ with $\alpha_{1}=-1$ and $q^{2}=x_{1}^{2}-x_{2}^{2}$ with $\alpha_{2}=1$.

Fix any $a_{1} \leq \alpha_{1} \neq \alpha_{2} \leq a_{2}$, then there are four double-cone solutions corresponding to each of $\alpha_{i}$. From these double-cone solutions we obtain eight more double-cone solutions, such that $\mathcal{S}_{1} \cap \mathcal{S}_{2}=\emptyset$, where $q^{i}, i=1,2$ can be either $q_{+}$or $q_{-}$corresponding to $\alpha_{i}$. The solution $u_{0}$ can be described graphycally as follows:

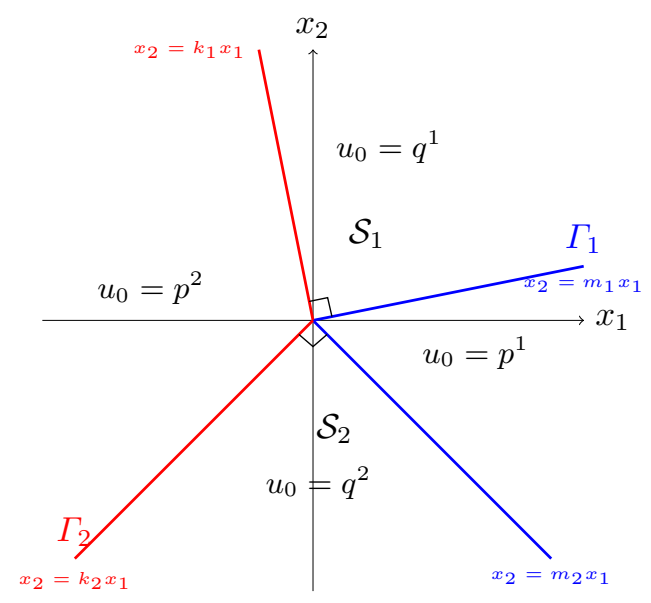

This is a general example of a double-cone solution (3.23), where the polynomial $q^{1}$, and the numbers $m_{1}, k_{1}$ correspond to $\alpha_{1}$, similarly $q^{2}$ and $m_{2}, k_{2}$ correspond to $\alpha_{2}$. We conclude that, if $u_{0}$ is a double-cone solution then the cone $\left\{u_{0}=p^{1}\right\}$ may have any opening angle $\theta, 0<\theta<\pi$, and the cone $\left\{u_{0}=p^{2}\right\}$ has an angle $\pi-\theta$. If $\theta=0$ or $\theta=\pi$, then $u_{0}$ is a halfspace solution corresponding to $p^{2}$ or $p^{1}$ respectively.

Finally, note that there are no homogeneous of degree two solutions $u_{0}$ corresponding to three or more different values of $\alpha$, since $u_{0}$ can have only an even number of $(q, \mathcal{S})$, and $\mathcal{S}$ always has an opening angle $\pi / 2$.

Case 2) If $\left(a_{1}+c_{2}\right)\left(a_{2}+c_{1}\right)<0$, then $c_{1}-a_{1}-c_{2}+a_{2} \neq 0$, and the equation (3.15) has a unique solution,

$$
\alpha=\frac{a_{2} c_{2}-a_{1} c_{1}}{c_{2}+a_{1}-a_{2}-c_{1}} .
$$

From the inequality $\left(a_{1}+c_{2}\right)\left(a_{2}+c_{1}\right)<0$ it easily follows that

$$
\max \left(a_{1},-c_{2}\right)<\alpha<\min \left(a_{2},-c_{1}\right) .
$$

Referring to (3.13), we can calculate

$$
\beta_{ \pm}= \pm \sqrt{\left(\alpha+c_{1}\right)\left(a_{1}-\alpha\right)}= \pm \frac{\sqrt{-\left(a_{2}-a_{1}\right)\left(c_{2}-c_{1}\right)\left(a_{2}+c_{1}\right)\left(a_{1}+c_{2}\right)}}{c_{2}+a_{1}-a_{2}-c_{1}} .
$$

According to (3.16),

$$
m_{ \pm}=\frac{\beta_{1,2}}{\alpha+c_{1}}=\mp \sqrt{-\frac{\left(c_{2}+a_{1}\right)\left(a_{2}-a_{1}\right)}{\left(a_{2}+c_{1}\right)\left(c_{2}-c_{1}\right)}}, \quad k_{ \pm}=\frac{\beta_{1,2}}{\alpha+c_{2}}= \pm \sqrt{-\frac{\left(c_{1}+a_{2}\right)\left(a_{2}-a_{1}\right)}{\left(a_{1}+c_{2}\right)\left(c_{2}-c_{1}\right)}} .
$$

Hence we obtain two harmonic polynomials $q_{+}$and $q_{-}$and four combinations of disjoint $\mathcal{S}_{1}$ and $\mathcal{S}_{2}$. Since in this case $\alpha$ is a fixed number, given by (3.28), there are only four double-cone solutions. 
Denote by $\vartheta_{i}$ the opening angle of the cone $\mathcal{S}_{i}$, then it follows from (3.30) that

$$
\begin{gathered}
\cos \vartheta_{i}= \pm \frac{1+k_{+} m_{+}}{\sqrt{1+\left(k_{+}\right)^{2}} \sqrt{1+\left(m_{+}\right)^{2}}}= \pm \frac{1+k_{-} m_{-}}{\sqrt{1+\left(k_{-}\right)^{2}} \sqrt{1+\left(m_{-}\right)^{2}}}= \\
= \pm \frac{\frac{c_{2}-c_{1}-a_{2}+a_{1}}{c_{2}-c_{1}}}{\sqrt{\frac{\left(a_{1}+c_{1}\right)\left(c_{2}-a_{2}+a_{1}-c_{1}\right)}{\left(a_{2}+c_{1}\right)\left(c_{2}-c_{1}\right)}} \sqrt{\frac{\left(a_{2}+c_{2}\right)\left(c_{2}-a_{2}+a_{1}-c_{1}\right)}{\left(a_{1}+c_{2}\right)\left(c_{2}-c_{1}\right)}}}= \pm \sqrt{\frac{\left(a_{1}+c_{2}\right)\left(a_{2}+c_{1}\right)}{\left(a_{1}+c_{1}\right)\left(a_{2}+c_{2}\right)}}, \text { for } i=1,2,
\end{gathered}
$$

hence $\vartheta_{1}=\vartheta_{2}=\vartheta$, and

$$
0<\cos ^{2} \vartheta<1 \text {. }
$$

In Example 3.4 $a_{1}=c_{1}=-1, a_{2}=2, c_{2}=0$, by a direct calculation we see that $\alpha=\frac{1}{2}$, $\beta= \pm \frac{\sqrt{3}}{2}$, and $\vartheta_{1}=\vartheta_{2}=\frac{\pi}{3}$ or $\vartheta_{1}=\vartheta_{2}=\frac{2 \pi}{3}$.

Case 3) If $\left(a_{1}+c_{2}\right)\left(c_{1}+a_{2}\right)>0$, then the statement follows from the inequality (3.17) in Lemma 3.7. Otherwise if $\left(a_{1}+c_{2}\right)\left(c_{1}+a_{2}\right)=0$, and $a_{1}+c_{2} \neq a_{2}+c_{1}$, there are only halfspace solutions. Indeed, assume that $a_{1}+c_{2}=0$, and $a_{2}+c_{1} \neq 0$, then $\alpha=a_{1}=-c_{2}$ by (3.28), and $\beta=0$. Hence we obtain that $u_{0}$ is a halfspace solution corresponding to $p^{1}$.

We say that a given polynomial $p$ has a sign, if $p \neq \equiv 0$, and $p \geq 0$ (or $p \leq 0$ ). Let us rephrase Theorem 3.8 in a more compact form.

Corollary 3.9. Let $p^{i}=a_{i} x_{1}^{2}+c_{i} x_{2}^{2}$ be given polynomials, satisfying (3.9) and (3.8). There exist double-cone solutions for the double obstacle problem with $p^{1}, p^{2}$, if and only if the following polynomial

$$
P(x)=P\left(x_{1}, x_{2}\right) \equiv p^{1}\left(x_{1}, x_{2}\right)+p^{2}\left(x_{2}, x_{1}\right)=\left(a_{1}+c_{2}\right) x_{1}^{2}+\left(c_{1}+a_{2}\right) x_{2}^{2}
$$

has no sign. If $P \equiv 0$, there are infinitely many double-cone solutions. If $P$ changes the sign, then there are four double-cone solutions, and if $P$ has a sign, there are none.

In other words, there exist double-cone solutions if and only if the matrix $D^{2} P$ is neither positive nor negative definite.

\subsection{Halfspace solutions}

Now we proceed to the discussion on the existence of halfspace solutions in $\mathbb{R}^{2}$, see Definition 3.1 . Let $u \in C^{1,1}$ be such that $w=u-p^{1}$ is a halfplane solution to the obstacle problem $\Delta w=$ $-\lambda_{1} \chi_{\{w>0\}}$, we need to check if $u \leq p^{2}$ in $\mathbb{R}^{2}$.

Lemma 3.10. Let $p^{i}=a_{i} x_{1}^{2}+c_{i} x_{2}^{2}$ be given polynomials, satisfying (3.9) and (3.8). Let $u^{1} \in C^{1,1}$ be a halfplane solution to the obstacle problem with obstacle $p^{1}$,

$$
u^{1}(x)= \begin{cases}q(x)=\alpha x_{1}^{2}+2 \beta x_{1} x_{2}-\alpha x_{2}^{2}>p^{1}(x), & \text { if } x_{2}>k x_{1} \\ p^{1}(x), & \text { if } x_{2} \leq k x_{1},\end{cases}
$$

where $\alpha, \beta$ and $k$ are real numbers. The function $u^{1}$ is a halfspace solution corresponding to $p^{1}$ (for the double obstacle problem with $p^{1}, p^{2}$ ), if and only if

$$
\min \left(-c_{1}, a_{2}\right) \geq \alpha \geq \max \left(a_{1},-c_{2}\right), \delta_{1}(\alpha):=a_{1} c_{1}-a_{2} c_{2}+\alpha\left(a_{1}-c_{1}-a_{2}+c_{2}\right) \leq 0,
$$


and

$$
\beta^{2}=-\left(\alpha-a_{1}\right)\left(\alpha+c_{1}\right), k=\frac{\beta}{\alpha+c_{1}} .
$$

Similarly, let $-u^{2} \in C^{1,1}$ be a halfplane solution to the obstacle problem with obstacle $-p^{2}$,

$$
u^{2}(x)= \begin{cases}q(x)=\alpha x_{1}^{2}+2 \beta x_{1} x_{2}-\alpha x_{2}^{2}<p^{2}(x), & \text { if } x_{2}>m x_{1} \\ p^{2}(x), & \text { if } x_{2} \leq m x_{1},\end{cases}
$$

Then $u^{2}$ is a halfplane solution corresponding to $p^{2}$, if and only if

$$
\min \left(-c_{1}, a_{2}\right) \geq \alpha \geq \max \left(a_{1},-c_{2}\right), \delta_{2}(\alpha):=-a_{1} c_{1}+a_{2} c_{2}-\alpha\left(a_{1}-c_{1}-a_{2}+c_{2}\right) \leq 0,
$$

and

$$
\beta^{2}=-\left(\alpha-a_{2}\right)\left(\alpha+c_{2}\right), m=\frac{\beta}{\alpha+c_{2}} .
$$

Proof. In order to show that $u^{1}$ is a halfspace solution (for the double obstacle problem) corresponding to $p^{1}$, we need to verify that $u^{1} \leq p^{2}$ in $\mathbb{R}^{2}$.

Let $t=\frac{x_{2}}{x_{1}}$, and consider the following polynomial

$$
f_{1}(t):=\frac{q(x)-p^{1}(x)}{x_{1}^{2}}=\left(-\alpha-c_{1}\right) t^{2}+2 \beta t+\left(\alpha-a_{1}\right) \geq 0 .
$$

Since $u^{1} \in C^{1,1}$, the polynomial $f_{1}$ has a double root at $t=k$, and therefore (3.34) holds, with $-c_{1} \geq \alpha \geq a_{1}$. Now let us prove (3.33). Consider the polynomial

$$
f_{2}(t):=\frac{p^{2}(x)-q(x)}{x_{1}^{2}}=\left(c_{2}+\alpha\right) t^{2}-2 \beta t+\left(a_{2}-\alpha\right) .
$$

Since $q \leq p^{2}$ on a halfplane, the polynomial $f_{2}$ has to be nonnegative. The latter is equivalent to the following inequalities

$$
a_{2} \geq \alpha \geq-c_{2} \text { and } \delta_{1}:=\beta^{2}-\left(a_{2}-\alpha\right)\left(c_{2}+\alpha\right) \leq 0 .
$$

Taking into account (3.34), we obtain the desired inequality (3.33).

The corresponding statement for $u^{2}$ can be proved similarly.

Theorem 3.11. (The existence of halfspace solutions)

Let the assumptions of Theorem 3.8 hold.

Case 1) If $a_{2}+c_{1}=c_{2}+a_{1}=0$, then there are infinitely many rotational invariant halfplane solutions corresponding to $p^{1}\left(p^{2}\right)$.

Case 2) If $\left(a_{1}+c_{2}\right)\left(c_{1}+a_{2}\right)<0$, then there exist infinitely many halfplane solutions corresponding to $p^{1}\left(p^{2}\right)$, and $\Gamma_{i}$ always remains inside a fixed cone, thus halfplane solutions are not rotationally invariant on the entire plane.

Case 3) $\left(a_{1}+c_{2}\right)\left(c_{1}+a_{2}\right) \geq 0$, and $a_{1}+c_{2} \neq 0$ or $a_{2}+c_{1} \neq 0$. If $a_{1}+c_{2} \geq 0, a_{2}+c_{1}>0$ then there are infinitely many rotationally invariant halfspace solutions corresponding to $p^{1}$, and at most two halfspace solutions corresponding to $p^{2}$. If $a_{1}+c_{2} \leq 0, a_{2}+c_{1}<0$ then there are infinitely many rotational invariant halfspace solutions corresponding to $p^{2}$, and at most two halfspace solutions corresponding to $p^{1}$. 
Proof. Case 1) In this case $\delta_{1}(\alpha)=\delta_{2}(\alpha)=0$ for any $\alpha$. By Lemma 3.10. $-c_{1} \geq \alpha \geq a_{1}$ can be any number, and $k(m)$ take any value in the closed interval $[-\infty, \infty]$. Hence $\Gamma_{1}\left(\Gamma_{2}\right)$ can be any line on the plane. Furthermore, if $u^{1}$ is a halfspace solution corresponding to $p^{1}$, then $\Gamma_{2}=\left\{u^{1}=p^{2}\right\}$ is a halfline, perpendicular to $\Gamma_{1}$.

Case 2) Without loss of generality we may assume that $a_{1}+c_{2}>0$ and $a_{2}+c_{1}<0$. Employing Lemma 3.10, (3.33) together with (3.8) and (3.9), we obtain the following chain of inequalities

$$
-c_{2}<a_{1} \leq \alpha \leq \frac{a_{2} c_{2}-a_{1} c_{1}}{a_{1}-c_{1}-a_{2}+c_{2}}<a_{2}<-c_{1}
$$

and therefore

$$
|k|=\sqrt{\frac{\alpha-a_{1}}{-\alpha-c_{1}}} \leq \sqrt{-\frac{\left(a_{2}-a_{1}\right)\left(a_{1}+c_{2}\right)}{\left(c_{2}-c_{1}\right)\left(a_{2}+c_{1}\right)}}:=K>0 .
$$

It follows from Lemma 3.10 that for any $\alpha$ satisfying (3.38) there are four halfspace solutions $u^{1}$ corresponding to $p^{1}$, and

$$
\Gamma_{1}=\partial\left\{u^{1}>p^{1}\right\}=\left\{x: x_{2}= \pm k x_{1}\right\} \subset\left\{x:\left|x_{2}\right| \leq K\left|x_{1}\right|\right\}
$$

by (3.39), and $\Gamma_{2}=\{0\}$ if $a_{1}<\alpha<\frac{a_{2} c_{2}-a_{1} c_{1}}{a_{1}-c_{1}-a_{2}+c_{2}}$.

Let us also discuss the halfspace solutions corresponding to $p^{2}$. According to Lemma 3.10. (3.36),

$$
-c_{2}<a_{1}<\frac{a_{2} c_{2}-a_{1} c_{1}}{a_{1}-c_{1}-a_{2}+c_{2}} \leq \alpha \leq a_{2}<-c_{1}
$$

and

$$
|m|=\sqrt{\frac{a_{2}-\alpha}{\alpha+c_{2}}} \leq \sqrt{-\frac{\left(a_{2}-a_{1}\right)\left(a_{2}+c_{1}\right)}{\left(c_{2}-c_{1}\right)\left(a_{1}+c_{2}\right)}}:=M>0 .
$$

Therefore we obtain infinitely many halfspace solutions $u^{2}$ corresponding to $p^{2}$, and

$$
\Gamma_{2}=\partial\left\{u^{2}>p^{2}\right\} \subset\left\{x:\left|x_{2}\right| \leq M\left|x_{1}\right|\right\} .
$$

Case 3) Assume that $a_{1}+c_{2} \geq 0$ and $c_{1}+a_{2} \geq 0$, then

$$
\begin{array}{r}
\delta_{1}=a_{1} c_{1}-a_{2} c_{2}+\alpha\left(a_{1}+c_{2}\right)-\alpha\left(a_{2}+c_{1}\right) \leq \\
a_{1} c_{1}-a_{2} c_{2}-c_{1}\left(a_{1}+c_{2}\right)-a_{1}\left(a_{2}+c_{1}\right)=-\left(a_{1}+c_{2}\right)\left(a_{2}+c_{1}\right) \leq 0,
\end{array}
$$

for any $\alpha$, such that $-c_{2} \leq a_{1} \leq \alpha \leq-c_{1} \leq a_{2}$. Hence there are infinitely many halfspace solutions corresponding to $p^{1}$, and $\Gamma_{1}$ can be any line on the plane (depending on $\alpha$ ). Next, assuming that $a_{1}+c_{2}>0$ and $c_{1}+a_{2}>0$, we show that there are no halfplane solutions corresponding to the upper obstacle $p^{2}$. Indeed, in this case

$$
\begin{array}{r}
\delta_{2}(\alpha)=-a_{1} c_{1}+a_{2} c_{2}-\alpha\left(a_{1}+c_{2}\right)+\alpha\left(a_{2}+c_{1}\right) \geq \\
-a_{1} c_{1}+a_{2} c_{2}+c_{1}\left(a_{1}+c_{2}\right)+a_{1}\left(a_{2}+c_{1}\right)=\left(a_{1}+c_{2}\right)\left(a_{2}+c_{1}\right)>0,
\end{array}
$$

and the statement follows from Lemma 3.10, (3.36). In this case $\Gamma_{2}=\{0\}$ for any halfspace solution $u^{1}$.

Finally, if $a_{1}+c_{2}>0$ but $c_{1}+a_{2}=0$, then $\alpha=-c_{1}=a_{2}$, and we obtain only two halfspace solutions corresponding to $p^{2}$.

If $a_{1}+c_{2}<0$ and $c_{1}+a_{2} \leq 0$, then we can consider the double obstacle problem with obstacles $-p^{2} \leq-p^{1}$, and see that there at most two halfspace solutions corresponding to $p^{1}$. 
Corollary 3.12. Under the assumptions of Theorems 3.8, 3.11 and Corollary 3.9 we have that;

Case 1) If $P \equiv 0$, there are infinitely many rotational invariant halfspace solutions corresponding to $p^{1}\left(p^{2}\right)$.

Case 2) $P$ changes the sign. There are infinitely many halfspace solutions corresponding to $p^{1}\left(p^{2}\right)$.

Case 3) $P$ has a sign, if $P(x) \geq 0$, there are infinitely many rotational invariant halfspace solutions corresponding to $p^{1}$, and at most two halfspace solutions corresponding to $p^{2}$. Similarly, if $P(x) \leq 0$, then are infinitely many rotational invariant halfspace solutions corresponding to $p^{2}$, and at most two halfspace solutions corresponding to $p^{1}$.

\section{Uniqueness of blow-ups, Case 1}

Let $u$ be a solution to the double obstacle problem (3.5), with polynomial obstacles $p^{1} \leq p^{2}$, satisfying $p^{1}(x)=p^{2}(x)$ iff $x=0$. We study the uniqueness of blow-ups of $u$ in Case 1 , i.e. when the polynomials $p^{i}$ are given by

$$
p^{1}(x)=-a x_{1}^{2}-c x_{2}^{2}, \quad p^{2}(x)=c x_{1}^{2}+a x_{2}^{2}, \text { where } a+c>0 .
$$

Consider the following harmonic polynomial $h(x):=\frac{-a+c}{2} x_{1}^{2}+\frac{a-c}{2} x_{2}^{2}$, then

$$
p^{1}(x)-h(x)=\frac{a+c}{2}\left(-x_{1}^{2}-x_{2}^{2}\right), \quad p^{2}(x)-h(x)=\frac{a+c}{2}\left(x_{1}^{2}+x_{2}^{2}\right) .
$$

Thus it is enough to study the uniqueness of blow-ups in the case

$$
p^{1}(x)=-x_{1}^{2}-x_{2}^{2}, \text { and } p^{2}(x)=x_{1}^{2}+x_{2}^{2} .
$$

From now on we study the solution $\frac{2(u-h)}{a+c}$ instead of $u$, but still call it $u$.

Let $r_{j} \rightarrow 0+$, as $j \rightarrow \infty$, and

$$
u_{0}(x):=\lim _{j \rightarrow \infty} \frac{u\left(r_{j} x\right)}{r_{j}^{2}}
$$

be a blow-up of $u$ at the origin. We know that there exists

$$
\lim _{r \rightarrow 0} W(u, r, 0)=\lim _{j \rightarrow \infty} W\left(u_{r_{j}}, 1,0\right) \equiv W\left(u_{0}, 1,0\right) .
$$

Hence if $\bar{u}_{0}$ is another blow-up solution, then $W\left(u_{0}, 1,0\right)=W\left(\bar{u}_{0}, 1,0\right)$. Denote by

$$
\mathcal{C}_{i}:=\left\{x=\left(x_{1}, x_{2}\right) \in \mathbb{R}^{2} ; u_{0}(x)=p^{i}(x)\right\},
$$

where $p^{i}$ are the polynomials in (4.1).

Let us calculate the values of $W\left(u_{0}, 1,0\right)$ for all the possible blow-up solutions $u_{0}$. By definition

$$
\begin{array}{r}
W\left(u_{0}, 1,0\right)=\int_{B_{1}}\left|\nabla u_{0}\right|^{2} d x-2 \int_{\partial B_{1}} u_{0}^{2} d S+2 \int_{B_{1}} \lambda_{1} u_{0} \chi_{\left\{u_{0}=p^{1}\right\}}+\lambda_{2} u_{0} \chi_{\left\{u_{0}=p^{2}\right\}} d x \\
=-\int_{B_{1}} u_{0} \Delta u_{0} d x+2 \int_{B_{1}} \lambda_{1} u_{0} \chi_{\left\{u_{0}=p^{1}\right\}}+\lambda_{2} u_{0} \chi_{\left\{u_{0}=p^{2}\right\}} d x \\
=\lambda_{1} \int_{B_{1} \cap \mathcal{C}_{1}} p^{1} d x+\lambda_{2} \int_{B_{1} \cap \mathcal{C}_{2}} p^{2} d x
\end{array}
$$


After substituting $\lambda_{1}=-4$ and $\lambda_{2}=4$ in (4.3), we obtain

$$
W\left(u_{0}, 1,0\right)=4 \int_{B_{1} \cap \mathcal{C}_{1}} r^{3} d r d \theta+4 \int_{B_{1} \cap \mathcal{C}_{2}} r^{3} d r d \theta
$$

and we may conclude from Theorem 3.8 that

$$
W\left(u_{0}, 1,0\right)= \begin{cases}0, & \text { if } u_{0} \text { is a harmonic second order polynomial } \\ 2 \pi, & \text { if } u_{0} \equiv p^{1} \text { or } u_{0} \equiv p^{2} \\ \pi, & \text { if } u_{0} \text { is a halfspace or a double-cone solution. }\end{cases}
$$

This gives three types of possible blow-ups at a fixed free boundary point.

Denote by

$$
u^{j}(x):=\frac{u\left(r_{j} x\right)}{r_{j}^{2}},
$$

and assume that

$$
u^{j} \rightarrow u_{0} \text { in } C^{1, \gamma}\left(B_{1}\right) .
$$

If $u_{0}$ is a polynomial or a halfspace solution to the double obstacle problem, then $u_{0}$ is a blow-up solution to a single obstacle problem. In this case the known techniques can be used to prove the uniqueness of blow-ups and to analyse the free boundary. We will provide a rigorous argument for that in the end of this section.

For now we focus on the case when $u_{0}$ is a double-cone solution. According to Theorem 3.8, $u_{0}$ can be described in terms of parameters $-1<\alpha_{1}, \alpha_{2}<1$. Let $\alpha_{i}=\cos \phi_{i}$, for some $0<\phi_{1}, \phi_{2}<\pi$. According to Lemma 3.10, $\beta_{i}= \pm \sqrt{1-\alpha_{i}^{2}}= \pm \sin \phi_{i}, m_{i}=\frac{\beta_{i}}{\cos \phi_{i}-1}=\mp \tan \frac{\phi_{i}}{2}$ and $k_{i}=\frac{\beta_{i}}{\cos \phi_{i}+1}= \pm \cot \frac{\phi_{i}}{2}$. Referring to (3.27), we see that

$$
\begin{array}{r}
q^{i}(r, \theta)=x_{1}^{2} \cos \phi_{i}-x_{2}^{2} \cos \phi_{i} \pm 2 x_{1} x_{2} \sin \phi_{i}=r^{2}\left(\cos \phi_{i} \cos ^{2} \theta-\cos \phi_{i} \sin ^{2} \theta \pm \sin 2 \theta \sin \phi_{1}\right) \\
=r^{2}\left(\cos \phi_{i} \cos 2 \theta \pm \sin 2 \theta \sin \phi_{i}\right)=r^{2} \cos \left(2 \theta \mp \phi_{i}\right) .
\end{array}
$$

Hence without loss of generality $u_{0}$ is the following function

$$
u_{0}=\mu=\mu_{\phi_{1}, \phi_{2}}(r, \theta):= \begin{cases}r^{2}, & \text { if }-\phi_{2} \leq 2 \theta \leq \phi_{1} \\ r^{2} \cos \left(2 \theta-\phi_{1}\right), & \text { if } \phi_{1} \leq 2 \theta \leq \pi+\phi_{1} \\ r^{2} \cos \left(2 \theta+\phi_{2}\right), & \text { if }-\pi-\phi_{2} \leq 2 \theta \leq-\phi_{2} \\ -r^{2}, & \text { otherwise. }\end{cases}
$$

For further analysis we need the following two easy lemmas.

Lemma 4.1. Let $u$ and $u_{0}$ be two solutions (with different boundary conditions) to the double obstacle problem in $B_{2} \subset \mathbb{R}^{n}$, with given obstacles $\psi^{1} \leq \psi^{2}$. Then for any $\zeta \in C_{0}^{2}\left(B_{2}\right)$ the following inequality holds

$$
\int_{B_{2}}\left(\nabla u-\nabla u_{0}\right) \cdot \nabla\left(\zeta^{2}\left(u-u_{0}\right)\right) d x \leq 0 .
$$

Furthermore,

$$
\left\|u-u_{0}\right\|_{W^{1,2}\left(B_{1}\right)} \leq C_{n}\left\|u-u_{0}\right\|_{L^{2}\left(B_{2}\right)},
$$

where $C_{n}$ is just a dimensional constant. 
Proof. The proof is quite standard. Given a solution $u$ to the double obstacle problem in $B_{1}$, then for any $\zeta \in C_{0}^{2}\left(B_{1}\right)$, the function $u_{t}(x):=u+t \zeta^{2}\left(u_{0}-u\right)$ is admissible for $t>0$ small enough depending only on $\zeta$. Hence

$$
\begin{aligned}
\int_{B_{2}}|\nabla u|^{2} d x \leq \int_{B_{2}}\left|\nabla u_{t}\right|^{2} d x=\int_{B_{2}}|\nabla u|^{2} d x & +2 t \int_{B_{2}} \nabla u \cdot \nabla\left(\zeta^{2}\left(u_{0}-u\right)\right) d x \\
& +t^{2} \int_{B_{2}}\left|\nabla\left(\zeta^{2}\left(u_{0}-u\right)\right)\right|^{2} d x
\end{aligned}
$$

after dividing the last inequality by $t>0$, and taking the limit as $t$ goes to zero, we obtain

$$
0 \leq \int_{B_{2}} \nabla u \cdot \nabla\left(\zeta^{2}\left(u_{0}-u\right)\right) d x=\int_{B_{2}}\left(u_{0}-u\right) \nabla u \cdot \nabla \zeta^{2} d x+\int_{B_{2}} \zeta^{2} \nabla u \cdot \nabla\left(u_{0}-u\right) d x .
$$

Similarly, the function $u_{0}+t \zeta^{2}\left(u-u_{0}\right)$ is admissible for the double obstacle problem, having solution $u_{0}$. Therefore

$$
0 \leq \int_{B_{2}} \nabla u_{0} \cdot \nabla\left(\zeta^{2}\left(u-u_{0}\right)\right) d x=\int_{B_{2}}\left(u-u_{0}\right) \nabla u_{0} \cdot \nabla \zeta^{2} d x+\int_{B_{1}} \zeta^{2} \nabla u_{0} \cdot \nabla\left(u-u_{0}\right) d x .
$$

The inequalities (4.10) and (4.11) together imply the inequality (4.8), and we proceed to the proof of the second statement in our lemma.

Choose $\zeta \in C_{0}^{2}\left(B_{3 / 2}\right)$, such that $0 \leq \zeta \leq 1$ and $\zeta \equiv 1$ in $B_{1}$. Combining the inequalities (4.10) and (4.11), we obtain

$$
\begin{array}{r}
\int_{B_{2}} \zeta^{2}\left|\nabla u-\nabla u_{0}\right|^{2} d x \leq-2 \int_{B_{2}} \zeta\left(u-u_{0}\right)\left(\nabla u-\nabla u_{0}\right) \cdot \nabla \zeta d x \\
\leq 2 \int_{B_{2}}|\nabla \zeta|^{2}\left(u-u_{0}\right)^{2} d x+\frac{1}{2} \int_{B_{2}} \zeta^{2}\left|\nabla u-\nabla u_{0}\right|^{2} d x,
\end{array}
$$

where we used Young's inequality in the last step. Hence

$$
\int_{B_{1}}\left|\nabla u-\nabla u_{0}\right|^{2} d x \leq \int_{B_{2}} \zeta^{2}\left|\nabla u-\nabla u_{0}\right|^{2} d x \leq 4 \int_{B_{2}}|\nabla \zeta|^{2}\left(u-u_{0}\right)^{2} d x \leq C_{n}\left\|u-u_{0}\right\|_{L^{2}\left(B_{2}\right)}^{2},
$$

where $C_{n}$ is just a dimensional constant, depending only on $\zeta$. The proof of the lemma is now complete.

Lemma 4.2. Let $\left\{u^{j}\right\}$ and $\left\{\mu^{j}\right\}$ be given sequences of solutions to the double obstacle problem in $B_{2}$. Assume that $\delta^{j}:=\left\|u^{j}-\mu^{j}\right\|_{L^{2}\left(B_{2}\right)} \rightarrow 0$, as $j \rightarrow \infty$. Define $v^{j}:=\frac{u^{j}-\mu^{j}}{\delta^{j}}$. Then up to a subsequence

$$
v^{j} \rightarrow v^{0} \text { weakly in } W^{1,2}\left(B_{1}\right) \text {, and } v^{j} \rightarrow v^{0} \text { in } L^{2}\left(B_{1}\right) \text {. }
$$

Furthermore, if $v^{0} \Delta v^{0}=0$ in a weak sense, then

$$
\left\|v^{j}-v^{0}\right\|_{W^{1,2}\left(B_{1 / 2}\right)} \rightarrow 0 \text {. }
$$

Proof. According to Lemma 4.1, $\left\|v^{j}\right\|_{W^{1,2}\left(B_{1}\right)} \leq C_{n}$, where $C_{n}$ is a dimensional constant. Hence (4.12) follows from the weak compactness of the space $W^{1,2}$ and from the Sobolev embedding theorem.

We will obtain the strong convergence in (4.13), if we show that

$$
\lim _{j \rightarrow \infty}\left\|\nabla v^{j}\right\|_{L^{2}\left(B_{1 / 2}\right)}=\left\|\nabla v^{0}\right\|_{L^{2}\left(B_{1 / 2}\right)} .
$$


According to Lemma 4.1] (4.8), for any $\zeta \in C_{0}^{2}\left(B_{1}\right)$ and for any $j$, the following inequality holds

$$
\int_{B_{2}} \nabla v^{j} \cdot \nabla\left(\zeta^{2} v^{j}\right) d x \leq 0
$$

Hence

$$
\begin{aligned}
\limsup _{j \rightarrow \infty} \int_{B_{1}} \zeta^{2}\left|\nabla v^{j}\right|^{2} & \leq-\lim _{j \rightarrow \infty} \int_{B_{1}} v^{j} \nabla v^{j} \cdot \nabla\left(\zeta^{2}\right)=-\int_{B_{1}} v^{0} \nabla v^{0} \cdot \nabla\left(\zeta^{2}\right) \\
& =\int_{B_{1}} \zeta^{2}\left|\nabla v^{0}\right|^{2}-\int_{B_{1}} \nabla v^{0} \cdot \nabla\left(\zeta^{2} v^{0}\right)=\int_{B_{1}} \zeta^{2}\left|\nabla v^{0}\right|^{2},
\end{aligned}
$$

where we used $v^{0} \Delta v^{0}=0$ in the last step. On the other hand we have that

$$
\liminf _{j \rightarrow \infty} \int_{B_{1}} \zeta^{2}\left|\nabla v^{j}\right|^{2} \geq \int_{B_{1}} \zeta^{2}\left|\nabla v^{0}\right|^{2},
$$

by the weak convergence $\zeta \nabla v^{j} \rightarrow \zeta \nabla v^{0}$ in $L^{2}\left(B_{1}\right)$. Choosing $\zeta \in C_{0}^{2}\left(B_{1}\right)$ such that $\zeta=1$ in $B_{1 / 2}$, we obtain $\left\|\nabla v^{j}-\nabla v^{0}\right\|_{L^{2}\left(B_{1 / 2}\right)} \rightarrow 0$.

Remark 4.3. The strong convergence $v^{j} \rightarrow v^{0}$ in $W^{1,2}\left(B_{1 / 2}\right)$ will not be used when proving the uniqueness of double-cone blow-up limits, but we will need it when discussing the uniqueness of halfspace blow-ups.

If $u^{j} \rightarrow u_{0}$, where $u_{0}$ is a homogeneous global solution, then $v^{0} \Delta v^{0}=0$ always holds, although in the statement of Lemma 4.1 we preferred to assume rather than prove it. We will prove that $v^{0} \Delta v^{0}=0$ in our later discussion.

Definition 4.4. Let $u$ be a solution to the double obstacle problem. We say that $u_{0}=\mu_{\phi_{1}, \phi_{2}}$ is a minimal double-cone solution with respect to $u$, if

$$
\left\|u-\mu_{\phi_{1}, \phi_{2}}\right\|_{L^{2}\left(B_{1}\right)} \leq\left\|u-\mu_{\phi_{1}+\tau, \phi_{2}+\delta}\right\|_{L^{2}\left(B_{1}\right)},
$$

for any $\tau, \delta$, such that $|\tau|<\pi-\phi_{1}$ and $|\delta|<\pi-\phi_{2}$.

It follows from Definition 4.4 that if $\mu_{\phi_{1}, \phi_{2}}$ is a minimal double-cone solution with respect to $u$, then

$$
\int_{\phi_{i} / 2}^{\pi / 2+\phi_{i} / 2} \int_{0}^{1} \sin \left(\phi_{i}-2 \theta\right)\left(u(r, \theta)-\mu_{\phi_{1}, \phi_{2}}(r, \theta)\right) r^{3} d r d \theta=0, \text { for } i=1,2 .
$$

We derive equation (4.19) by taking the partial derivatives of $\left\|u-\mu_{\phi_{1}+\tau, \phi_{2}+\delta}\right\|_{L^{2}\left(B_{1}\right)}$ at the origin with respect to variables $\tau$ and $\delta$.

Proposition 4.5. Let $u^{j}$ be a sequence of solutions to the double obstacle problem with obstacles $p^{1}(x)=-x_{1}^{2}-x_{2}^{2}$ and $p^{1}(x)=x_{1}^{2}+x_{2}^{2}$ in $\Omega \subset \mathbb{R}^{2}, B_{2} \subset \subset \Omega$. Assume that (4.6) holds, where $u_{0}=\mu$ is given by (4.7). Denote by

$$
v^{j}(x):=\frac{u^{j}(x)-\mu^{j}(x)}{\left\|u^{j}-\mu^{j}\right\|_{L^{2}\left(B_{2}\right)}},
$$

where $\mu^{j}$ is a minimal double-cone solution with respect to $u^{j}$. Then (4.12) holds up to a subsequence, where $v^{0} \equiv 0$ in $\mathcal{C}_{1} \cup \mathcal{C}_{2}$, and $\Delta v^{0}=0$ in each of the components of the noncoincidence set $\Omega_{12}=\mathcal{S}_{1} \cup \mathcal{S}_{2}$, where the cones $\mathcal{C}_{i}$ and $\mathcal{S}_{i}$ correspond to $\mu$. Furthermore, it follows from the minimality assumption that

$$
\int_{0}^{1} \int_{\phi_{i} / 2}^{\pi / 2+\phi_{i} / 2} v^{0}(r, \theta) \sin \left(2 \theta-\phi_{i}\right) d \theta r^{3} d r=0 \text { for } i=1,2 .
$$


Proof. It follows from the minimality assumption and from the triangle inequality, that $\mu^{j} \rightarrow \mu$;

$$
\left\|\mu^{j}-\mu\right\|_{L^{2}\left(B_{1}\right)} \leq\left\|u^{j}-\mu^{j}\right\|_{L^{2}\left(B_{1}\right)}+\left\|u^{j}-\mu\right\|_{L^{2}\left(B_{1}\right)} \leq 2\left\|u^{j}-\mu\right\|_{L^{2}\left(B_{1}\right)} \rightarrow 0 .
$$

We show that for any $K \subset \subset \mathcal{C}_{1} \cap B_{1}$, the functions $v^{j}$ vanish in $K$ for large $j$, since then we may conclude that $v^{0} \equiv 0$ in $\mathcal{C}_{1}$. Let $K \subset \subset V \subset \subset \mathcal{C}_{1}$, and $d:=\operatorname{dist}(K, \partial V)$. It follows from (4.6) that for any $\varepsilon>0$ there exists $j(\varepsilon)$, such that $\left|u^{j}(x)-p^{1}(x)\right| \leq \varepsilon$, for any $x \in K$, provided $j \geq j(\varepsilon)$ is large enough, depending only on $\varepsilon$. Take $0<\varepsilon<\frac{d^{2}}{4}$. Let us denote by $w^{j}:=u^{j}-p^{1}$, then $0 \leq w^{j} \leq \varepsilon$ solves the following normalized obstacle problem with zero obstacle

$$
\Delta w^{j}=-\lambda_{1} \chi_{\left\{w^{j}>0\right\}}=4 \chi_{\left\{w^{j}>0\right\}} \text { in } K .
$$

Fix $x_{0} \in K$, if $w_{j}\left(x_{0}\right)>0$, then we can apply the maximum growth lemma (Lemma 5 in [4) for the solution to the classical obstacle problem, and obtain

$$
\frac{d^{2}}{4}>\varepsilon \geq \sup _{B_{d}\left(x_{0}\right)} w^{j} \geq \frac{d^{2}}{2}
$$

which is not possible, therefore $w^{j}\left(x_{0}\right)=0$. Hence we may conclude that for $j>>1$ large, $v^{j}$ is vanishing in $K$, for any $K \subset \subset \mathcal{C}_{1} \cap B_{1}$.

Let $U \subset \subset \mathcal{S}_{1} \cap B_{1}$ be any open set, then $p^{1}<u^{j}<p^{2}$ and $p^{1}<\mu^{j}<p^{2}$ for large $j$, hence $\Delta v^{j}=0$, and after passing to the limit as $j \rightarrow \infty$, we obtain $\Delta v^{0}=0$ in $\mathcal{S}_{1} \cap B_{1}$. Hence $v^{0}$ is a harmonic function outside its support, and $v^{j} \rightarrow v^{0}$ in $W^{1,2}\left(B_{1 / 2}\right)$. $u^{j}$.

We obtain (4.21) by passing to the limit as $j \rightarrow \infty$ in equation (4.19) applied for the solutions

Lemma 4.6. Let $v^{0}$ be the function in Proposition 4.5, then

$$
\left\|v^{0}(s x)\right\|_{L^{2}\left(B_{1}\right)} \leq s^{4}\left\|v^{0}\right\|_{L^{2}\left(B_{1}\right)},
$$

for any $0<s<1$.

Proof. According to Proposition 4.5 $v^{0}$ is a harmonic function in the sector

$$
\mathcal{S}_{1} \cap B_{1}=\left\{\phi_{1} \leq 2 \theta \leq \phi_{1}+\pi\right\} \cap B_{1},
$$

and $v^{0}$ satisfies the following boundary conditions in the trace sense;

$$
v^{0}\left(r, \phi_{1} / 2\right)=v^{0}\left(r, \phi_{1} / 2+\pi / 2\right)=0, \text { for all } 0<r<1 .
$$

Therefore

$$
v^{0}(r, \theta)=\sum_{k=1}^{\infty} r^{2 k}\left(A_{k} \cos (2 k \theta)+B_{k} \sin (2 k \theta)\right) \quad \text { in } \mathcal{S}_{1} \cap B_{1},
$$

and according to (4.24), we have that

$$
A_{k} \cos k \phi_{1}+B_{k} \sin k \phi_{1}=0, \text { for } k=1,2, \ldots
$$

We claim that (4.26) implies

$$
\int_{\phi_{1} / 2}^{\pi / 2+\phi_{1} / 2}\left(A_{k} \cos (2 k \theta)+B_{k} \sin (2 k \theta)\right) \sin \left(2 \theta-\phi_{1}\right) d \theta=0, \text { for all } k=2,3, \ldots
$$


The proof of (4.27) is straightforward. Fix $k \geq 2$, and assume that $\sin k \phi_{1} \neq 0$, then

$$
B_{k}=-A_{k} \frac{\cos k \phi_{1}}{\sin k \phi_{1}}
$$

hence we obtain

$$
\begin{array}{r}
\int_{\phi_{1} / 2}^{\pi / 2+\phi_{1} / 2}\left(A_{k} \cos (2 k \theta)+B_{k} \sin (2 k \theta)\right) \sin \left(2 \theta-\phi_{1}\right) d \theta \\
=\frac{A_{k}}{\sin k \phi_{1}} \int_{\phi_{1} / 2}^{\pi / 2+\phi_{1} / 2}\left(\cos (2 k \theta) \sin k \phi_{1}-\cos k \phi_{1} \sin (2 k \theta)\right) \sin \left(2 \theta-\phi_{1}\right) d \theta \\
=\frac{-A_{k}}{\sin k \phi_{1}} \int_{\phi_{1} / 2}^{\pi / 2+\phi_{1} / 2} \sin \left(k\left(2 \theta-\phi_{1}\right)\right) \sin \left(2 \theta-\phi_{1}\right) d \theta=\frac{-A_{k}}{2 \sin k \phi_{1}} \int_{0}^{\pi} \sin k t \sin t d t=0, \quad \text { if } k \neq 1 .
\end{array}
$$

If $\sin k \phi_{1}=0$, then $\cos k \phi_{1} \neq 0$, and the proof of (4.27) works similarly.

In the next step we show that $A_{1}=B_{1}=0$. By using the orthogonality property (4.21) and (4.27), and employing elementary trigonometric identities, we obtain

$$
\begin{array}{r}
0=\int_{0}^{1} \int_{\phi_{1} / 2}^{\pi / 2+\phi_{1} / 2} v^{0}(r, \theta) \sin \left(2 \theta-\phi_{1}\right) d \theta r^{3} d r \\
=\int_{0}^{1} \int_{\phi_{1} / 2}^{\pi / 2+\phi_{1} / 2} \sum_{k=1}^{\infty} r^{2 k+3}\left(A_{k} \cos (2 k \theta)+B_{k} \sin (2 k \theta)\right) \sin \left(2 \theta-\phi_{1}\right) d \theta d r \\
=\sum_{k=1}^{\infty} \frac{1}{2(k+2)} \int_{\phi_{1} / 2}^{\pi / 2+\phi_{1} / 2}\left(A_{k} \cos (2 k \theta)+B_{k} \sin (2 k \theta)\right) \sin \left(2 \theta-\phi_{1}\right) d \theta \\
=\frac{1}{4} \int_{\phi_{1} / 2}^{\pi / 2+\phi_{1} / 2}\left(A_{1} \cos (2 \theta)+B_{1} \sin (2 \theta)\right) \sin \left(2 \theta-\phi_{1}\right) d \theta \\
=\frac{1}{4} \int_{\phi_{1} / 2}^{\pi / 2+\phi_{1} / 2}-A_{1} \sin \phi_{1} \cos ^{2} 2 \theta+B_{1} \cos \phi_{1} \sin ^{2} 2 \theta d \theta=\frac{\pi}{16}\left(-A_{1} \sin \phi_{1}+B_{1} \cos \phi_{1}\right) .
\end{array}
$$

Hence

$$
-A_{1} \sin \phi_{1}+B_{1} \cos \phi_{1}=0 .
$$

On the other hand

$$
A_{1} \cos \phi_{1}+B_{1} \sin \phi_{1}=0
$$

which is (4.26) for $k=1$. It is easy to see that (4.28) together with (4.29) imply $A_{1}=B_{1}=0$. By (4.25),

$$
v^{0}(s x)=v^{0}(s r, \theta)=s^{4} \sum_{k=2}^{+\infty} s^{2(k-2)} r^{2 k}\left(A_{k} \cos (2 k \theta)+B_{k} \sin (2 k \theta)\right) \text { in } \mathcal{S}_{1} \cap B_{1} .
$$

Hence we obtain

$$
\left\|v^{0}(s x)\right\|_{L^{2}\left(\mathcal{S}_{1} \cap B_{1}\right)} \leq s^{4}\left\|v^{0}\right\|_{L^{2}\left(\mathcal{S}_{1} \cap B_{1}\right)} .
$$

Analogously,

$$
\left\|v^{0}(s x)\right\|_{L^{2}\left(\mathcal{S}_{2} \cap B_{1}\right)} \leq s^{4}\left\|v^{0}\right\|_{L^{2}\left(\mathcal{S}_{2} \cap B_{1}\right)} .
$$

According to Proposition 4.5, $v_{0} \equiv 0$ in $B_{1} \backslash\left(\mathcal{S}_{1} \cup \mathcal{S}_{2}\right)$, hence

$$
\left\|v^{0}(s x)\right\|_{L^{2}\left(B_{1}\right)}=\left\|v^{0}(s x)\right\|_{L^{2}\left(\mathcal{S}_{1} \cap B_{1}\right)}+\left\|v^{0}(s x)\right\|_{L^{2}\left(\mathcal{S}_{2} \cap B_{1}\right)} .
$$


The desired inequality, (4.23), now follows from (4.30) and (4.31).

Corollary 4.7. For any $\varepsilon>0$ and $0<s<1$, there exists $\delta=\delta(\varepsilon, s)$ such that if

$$
\left\|u-\mu_{0}\right\|_{L^{2}\left(B_{2}\right)} \leq \delta
$$

then

$$
\left\|u_{s}-\mu_{0}\right\|_{L^{2}\left(B_{1}\right)} \leq s\left\|u-\mu_{0}\right\|_{L^{2}\left(B_{1}\right)}+\varepsilon\left\|u-\mu_{0}\right\|_{L^{2}\left(B_{2}\right)},
$$

where $\mu_{0}$ is a minimal double-cone solution with respect to $u$.

Proof. We argue by contradiction. Let $u^{j}$ be a sequence of solutions to the double obstacle problem and assume that

$$
\left\|u^{j}-\mu^{j}\right\|_{L^{2}\left(B_{1}\right)}:=\delta_{j} \rightarrow 0,
$$

but there exist $0<s<1$ and $\varepsilon>0$, such that

$$
\left\|u_{s}^{j}-\mu^{j}\right\|_{L^{2}\left(B_{1}\right)}>s\left\|u^{j}-\mu^{j}\right\|_{L^{2}\left(B_{1}\right)}+\varepsilon \delta_{j} .
$$

Let $v^{j}$ be the sequence defined by (4.20), then by (4.33)

$$
\left\|v_{s}^{j}\right\|_{L^{2}\left(B_{1}\right)}>s\left\|v^{j}\right\|_{L^{2}\left(B_{1}\right)} \text { and }\left\|v^{j}\right\|_{L^{2}\left(B_{1}\right)}>\varepsilon .
$$

Applying Proposition 4.5 we may pass to the limit in (4.34) as $j \rightarrow \infty$, and obtain

$$
\left\|v_{s}^{0}\right\|_{L^{2}\left(B_{1}\right)} \geq s\left\|v^{0}\right\|_{L^{2}\left(B_{1}\right)} \text { and }\left\|v^{0}\right\|_{L^{2}\left(B_{1}\right)} \geq \varepsilon,
$$

hence

$$
\left\|v_{s}^{0}\right\|_{L^{2}\left(B_{1}\right)} \geq s\left\|v^{0}\right\|_{L^{2}\left(B_{1}\right)}>s^{2}\left\|v^{0}\right\|_{L^{2}\left(B_{1}\right)},
$$

and we derive a contradiction to Lemma 4.6

Assume that $u_{0}=\mu$ given by (4.7) is a blow-up for $u$ at the origin, that is (4.5) and (4.6) hold for a sequence $r_{j} \rightarrow 0$. We want to show that the blow-up of $u$ at the origin is unique;

$$
u_{r}=\frac{u(r x)}{r^{2}} \rightarrow u_{0}(x), \text { as } r \rightarrow 0
$$

and describe the rate of convergence $u_{r} \rightarrow u_{0}$ as $r \rightarrow 0+$.

Proposition 4.8. Let $u$ be the solution to the double obstacle problem in $\Omega, B_{2} \subset \subset \Omega \subset \mathbb{R}^{2}$, with obstacles $p^{1}(x)=-x_{1}^{2}-x_{2}^{2}$ and $p^{2}(x)=x_{1}^{2}+x_{2}^{2}$. Assume that $\|u-\mu\|_{L^{2}\left(B_{2}\right)}=\delta$ is small, where $\mu$ is a double-cone solution, then there exists a double-cone solution $u_{0}$, such that $u_{r} \rightarrow u_{0}$. Furthermore, for any $0<\gamma<1$,

$$
\left\|u_{r}-u_{0}\right\|_{L^{2}\left(B_{1}\right)} \leq C_{n} r^{\gamma}\|u-\mu\|_{L^{2}\left(B_{2}\right)}
$$

provided $\delta>0$ is small depending on $\gamma$.

Proof. Let $\frac{1}{4} \leq s<\frac{1}{2}$ be a fixed number, and $\tau:=s^{\gamma}>s$. We use an induction argument to show that for $\delta>0$ small enough

$$
\left\|u_{s^{k+1}}-\mu^{k}\right\|_{L^{2}\left(B_{1}\right)} \leq \tau^{k+1} \delta, \text { and }\left\|\mu^{k+1}-\mu^{k}\right\|_{L^{2}\left(B_{1}\right)} \leq 2 \tau^{k+1} \delta, \quad k=0,1,2,3, \ldots
$$


where by definition $\mu^{k}$ is a minimal double-cone solution with respect to $u_{s^{k}}$.

Let us show that (4.36) is true for $k=0$. First we observe that by the triangle inequality and the minimality assumption,

$$
\left\|\mu-\mu^{0}\right\|_{L^{2}\left(B_{1}\right)} \leq\|u-\mu\|_{L^{2}\left(B_{1}\right)}+\left\|u-\mu^{0}\right\|_{L^{2}\left(B_{1}\right)} \leq 2\|u-\mu\|_{L^{2}\left(B_{1}\right)} .
$$

Note that since $\mu^{k}$ are homogeneous of degree two functions, the following relation is true

$$
\left\|\mu^{k+1}-\mu^{k}\right\|_{L^{2}\left(B_{2}\right)}=8\left\|\mu^{k+1}-\mu^{k}\right\|_{L^{2}\left(B_{1}\right)}, \text { for all } k=0,1,2, \ldots .
$$

Now let us proceed to the proof of (4.36) for $k=0$. According to Corollary 4.7 and (4.37),

$$
\begin{array}{r}
\left\|u_{s}-\mu_{0}\right\|_{L^{2}\left(B_{1}\right)} \leq s\left\|u-\mu^{0}\right\|_{L^{2}\left(B_{1}\right)}+\varepsilon\left\|u-\mu^{0}\right\|_{L^{2}\left(B_{2}\right)} \leq s\|u-\mu\|_{L^{2}\left(B_{1}\right)} \\
+\varepsilon\|u-\mu\|_{L^{2}\left(B_{2}\right)}+\varepsilon\left\|\mu-\mu^{0}\right\|_{L^{2}\left(B_{2}\right)} \leq s\|u-\mu\|_{L^{2}\left(B_{1}\right)}+\varepsilon\|u-\mu\|_{L^{2}\left(B_{2}\right)} \\
+16 \varepsilon\|u-\mu\|_{L^{2}\left(B_{1}\right)} \leq(s+17 \varepsilon)\|u-\mu\|_{L^{2}\left(B_{2}\right)} \leq \tau\|u-\mu\|_{L^{2}\left(B_{2}\right)},
\end{array}
$$

where we take $0<\varepsilon<\frac{\tau-s}{17}$. Thus we obtain $\left\|u_{s}-\mu^{0}\right\|_{L^{2}\left(B_{1}\right)} \leq \tau\|u-\mu\|_{L^{2}\left(B_{2}\right)}$, hence

$$
\left\|\mu^{1}-\mu^{0}\right\|_{L^{2}\left(B_{1}\right)} \leq\left\|u_{s}-\mu^{1}\right\|_{L^{2}\left(B_{1}\right)}+\left\|u_{s}-\mu^{0}\right\|_{L^{2}\left(B_{1}\right)} \leq 2\left\|u_{s}-\mu^{0}\right\|_{L^{2}\left(B_{1}\right)} \leq 2 \tau\|u-\mu\|_{L^{2}\left(B_{2}\right)},
$$

which completes the proof of (4.36) for $k=0$.

Let us assume (4.36) holds up to and including $k$, we will show that (4.36) holds for $k+1$. First note that $\left\|u_{s^{k+1}}-\mu^{k+1}\right\|_{L^{2}\left(B_{2}\right)}$ is small. Indeed, since $1 / 4<s<1 / 2$, we obtain

$$
\begin{array}{r}
\left\|u_{s^{k+1}}-\mu^{k}\right\|_{L^{2}\left(B_{2}\right)}=\frac{1}{s^{2}}\left\|u_{s^{k}}-\mu^{k}\right\|_{L^{2}\left(B_{2 s}\right)} \leq 16\left\|u_{s^{k}}-\mu^{k}\right\|_{L^{2}\left(B_{1}\right)} \\
\leq 16\left\|u_{s^{k}}-\mu^{k-1}\right\|_{L^{2}\left(B_{1}\right)} \leq 16 \tau^{k} \delta \leq 16 \delta
\end{array}
$$

by the induction assumption. According to Corollary 4.7 for any $\varepsilon>0$, we can choose $16 \delta>0$ to be small depending on $\varepsilon$ and $s$, and obtain

$$
\begin{aligned}
& \left\|u_{s^{k+2}}-\mu^{k+1}\right\|_{L^{2}\left(B_{1}\right)} \leq s\left\|u_{s^{k+1}}-\mu^{k+1}\right\|_{L^{2}\left(B_{1}\right)}+\varepsilon\left\|u_{s^{k+1}}-\mu^{k+1}\right\|_{L^{2}\left(B_{2}\right)} \\
& \leq s\left\|u_{s^{k+1}}-\mu^{k}\right\|_{L^{2}\left(B_{1}\right)}+\varepsilon\left\|u_{s^{k+1}}-\mu^{k}\right\|_{L^{2}\left(B_{2}\right)}+\varepsilon\left\|\mu^{k+1}-\mu^{k}\right\|_{L^{2}\left(B_{2}\right)} \\
& \leq s\left\|u_{s^{k+1}}-\mu^{k}\right\|_{L^{2}\left(B_{1}\right)}+16 \varepsilon\left\|u_{s^{k}}-\mu^{k-1}\right\|_{L^{2}\left(B_{1}\right)}+8 \varepsilon\left\|\mu^{k+1}-\mu^{k}\right\|_{L^{2}\left(B_{1}\right)},
\end{aligned}
$$

where we used (4.39) and (4.38) in the last step. Recalling our induction assumption, we obtain

$$
\left\|u_{s^{k+2}}-\mu^{k+1}\right\|_{L^{2}\left(B_{1}\right)} \leq\left(s \tau^{k+1}+16 \varepsilon \tau^{k}+8 \varepsilon \tau^{k+1}\right) \delta \leq \tau^{k+2} \delta,
$$

by choosing $\varepsilon<\frac{\tau(\tau-s)}{16+8 \tau}$. It follows from the triangle inequality and the definition of minimal double-cone solutions that

$$
\begin{array}{r}
\left\|\mu^{k+2}-\mu^{k+1}\right\|_{L^{2}\left(B_{1}\right)} \leq\left\|u_{s^{k+2}}-\mu^{k+2}\right\|_{L^{2}\left(B_{1}\right)}+\left\|u_{s^{k+2}}-\mu^{k+1}\right\|_{L^{2}\left(B_{1}\right)} \\
\leq 2\left\|u_{s^{k+2}}-\mu^{k+1}\right\|_{L^{2}\left(B_{1}\right)} \leq 2 \tau^{k+2} \delta .
\end{array}
$$

The proof of the inequalities (4.36) is therefore complete.

Now we are ready to show that $\mu^{k}$ is a Cauchy sequence, and therefore converges. For any $m, k \in \mathbb{N}$

$$
\left\|\mu^{k+m}-\mu^{k}\right\|_{L^{2}\left(B_{1}\right)} \leq \sum_{l=k}^{k+m-1}\left\|\mu^{l+1}-\mu^{l}\right\|_{L^{2}\left(B_{1}\right)} \leq \sum_{l=k}^{k+m-1} \tau^{l+1} \delta \leq \frac{\tau^{k+1}}{1-\tau} \delta \rightarrow 0,
$$


independent of $m$. Hence there exists $u_{0}$, such that $\mu^{k} \rightarrow u_{0}$, furthermore

$$
\left\|\mu^{k}-u_{0}\right\|_{L^{2}\left(B_{1}\right)} \leq \frac{\tau^{k+1}}{1-\tau} \delta .
$$

The inequalities (4.36) and (4.41) together with the triangle inequality imply that

$$
\left\|u_{s^{k}}-u_{0}\right\|_{L^{2}\left(B_{1}\right)} \leq 2 \tau^{k} \delta .
$$

Finally let us observe that for any $0<r<1$ there exists a nonnegative integer $k$ such that $s^{k+1} \leq r<s^{k}$. Hence

$$
\left\|u_{r}-u_{0}\right\|_{L^{2}\left(B_{1}\right)} \leq s^{-3}\left\|u_{s^{k}}-u_{0}\right\|_{L^{2}\left(B_{1}\right)} \leq 2 \cdot 4^{3} \tau^{k} \delta \leq 4^{4} r^{\gamma} \delta,
$$

where $\gamma=\frac{\ln \tau}{\ln s}<1$.

Corollary 4.9. Assume that $\mu$ given by (4.7) is a blow-up for $u$ at the origin, that is (4.5) and (4.6) hold for a sequence $r_{j} \rightarrow 0$. Then the blow-up of $u$ at the origin is unique;

$$
\frac{u(r x)}{r^{2}} \rightarrow \mu(x), \text { as } r \rightarrow 0 .
$$

Proof. Since $u_{r_{j}} \rightarrow \mu$ as $j \rightarrow \infty$, for any $\delta>0$ small we can find a small $\rho>0$ such that $\left\|u_{\rho}-\mu\right\|_{L^{2}\left(B_{2}\right)} \leq \delta$. Now we can apply Proposition 4.8 for the function $u_{\rho}$, and obtain $u_{\rho r} \rightarrow u_{0}$ as $r \rightarrow 0+$. Hence $u_{r} \rightarrow u_{0}$ and $u_{0}=\mu$.

Theorem 4.10. Let $u$ be the solution to the two-dimensional double obstacle problem with obstacles $p^{1}=-x_{1}^{2}-x_{2}^{2}$ and $p^{2}=x_{1}^{2}+x_{2}^{2}$. Assume that $\|u-\mu\|_{L^{2}\left(B_{2}\right)}=\delta$ is sufficiently small, where $\mu$ is a double-cone solution. Then in a small ball $B_{r_{0}}$ the free boundary consists of four $C^{1, \gamma}$ - graphs meeting at the origin, denoted by $\Gamma_{1}^{+}, \Gamma_{1}^{-}, \Gamma_{2}^{+}, \Gamma_{2}^{-}$. Neither $\Gamma_{1}=\Gamma_{1}^{+} \cup \Gamma_{1}^{-}$nor $\Gamma_{2}=\Gamma_{2}^{+} \cup \Gamma_{2}^{-}$has a normal at the origin. The curves $\Gamma_{1}^{+}$and $\Gamma_{2}^{+}$cross at a right angle, the same is true for $\Gamma_{1}^{-}$and $\Gamma_{2}^{-}$.

Proof. The proof of the theorem is based on Proposition 4.8 and on similar estimates obtained for the classical obstacle problem in [2]. According to Proposition 4.8 there exists a double-cone solution $u_{0}$ such that (4.35) holds. Moreover, applying Lemma 4.1 we obtain

$$
\left\|u_{r}-u_{0}\right\|_{W^{1,2}\left(B_{1 / 2}\right)} \leq C r^{\gamma}\|u-\mu\|_{L^{2}\left(B_{2}\right)} .
$$

Without loss of generality we assume that $u_{0}$ is given by (4.7); that is

$$
u_{0}=\mu_{\phi_{1}, \phi_{2}}(r, \theta):= \begin{cases}r^{2}, & \text { if }-\phi_{2} \leq 2 \theta \leq \phi_{1} \\ r^{2} \cos \left(\phi_{1}-2 \theta\right), & \text { if } \phi_{1} \leq 2 \theta \leq \pi+\phi_{1} \\ r^{2} \cos \left(\phi_{2}+2 \theta\right), & \text { if }-\pi-\phi_{2} \leq 2 \theta \leq-\phi_{2} \\ -r^{2}, & \text { otherwise }\end{cases}
$$

As before, we denote by $\mathcal{C}_{i}=\left\{u_{0}=p^{i}\right\}$, and let $\vartheta_{i}$ be the opening angle for $\mathcal{C}_{i}$, then $0<\vartheta_{i}<\pi, \vartheta_{1}=\left(\phi_{1}+\phi_{2}\right) / 2$ and $\vartheta_{2}=\pi-\vartheta_{1}$.

We want to show the regularity of $\Gamma_{2}=\partial\left\{u=p^{2}\right\}$ in a neighbourhood of the origin. We perform the proof in two steps. 


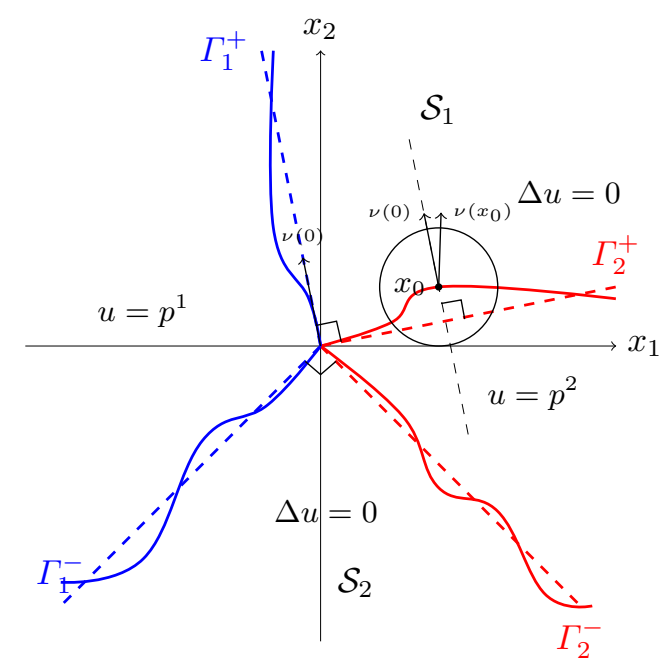

Figure 4.1: The local behaviour of the free boundary, with obstacles touching at a single point in Case 1 . Here $\Gamma_{i}^{ \pm}$are the pieces of the free boundary for $u$, while the dashed lines are the free boundary to the double-cone solution $u_{0}$.

Step 1: We show that $\Gamma_{2} \cap B_{r_{0}} \subset \overline{(Q \backslash K)}$, for any open cones $Q$ and $K$, having a common vertex at the origin, such that $K \subset \mathcal{C}_{2} \subset \bar{Q}$ and $\partial K \cap \partial \mathcal{C}_{2}=\partial Q \cap \partial \mathcal{C}_{2}=\{0\}$, where $r_{0}>0$ is small depending on $K, Q$.

Let $K \subset \mathcal{C}_{2}$ be a cone with a vertex at the origin, such that $\partial K \cap \partial \mathcal{C}_{2}=\{0\}$. Fix $0<\varrho<1 / 8$, and denote by $V:=K \cap\{\varrho<x<1 / 2\}$, and $\sigma:=\operatorname{dist}\left(V, \partial \mathcal{C}_{2}\right)$. First we will show that $u_{r}(x)=p^{2}(x)$ in $V$ for small $r>0$. Take $0<\varepsilon<\frac{\sigma^{2}}{4}$, then there exists $r_{\varepsilon}=r_{\sigma}$, such that $\left|u_{r}(x)-u_{0}(x)\right| \leq \varepsilon$ if $r \leq r_{\varepsilon}$. Let $\omega:=p^{2}-u_{r}$, for a fixed $r<r_{\varepsilon}$, then $0 \leq \omega \leq \varepsilon$ solves the following normalised obstacle problem with zero obstacle,

$$
\Delta \omega=\lambda_{2} \chi_{\{\omega>0\}} \text { in } \mathcal{C}_{2} .
$$

Fix $x_{0} \in V$, if $\omega\left(x_{0}\right)>0$, then we can apply the maximum growth lemma (Lemma 5 in [4]) for the solution to the obstacle problem, and obtain

$$
\frac{\sigma^{2}}{4}>\varepsilon \geq \sup _{B_{\sigma}\left(x_{0}\right)} \omega \geq \frac{\sigma^{2}}{2},
$$

which is not possible, hence $\omega\left(x_{0}\right)=0$.

Thus we have shown that $\frac{u(r x)}{r^{2}}=p^{2}(x)$ for all $r<r_{\varepsilon}$ and any $x \in K$, such that $\varrho<|x|<1 / 2$. Hence $u(y)=p^{2}(y)$ if $\varrho r<|y|<\frac{r}{2}$ for all $r<r_{\varepsilon}$, and therefore $u=p^{2}$ in $K \cap B_{r_{0}}, r_{0}:=r_{\varepsilon} / 2$.

Taking another open cone $Q$, with a vertex at the origin, and such that $\mathcal{C}_{2} \subset Q, \partial Q \cap \partial \mathcal{C}_{2}=$ $\{0\}$, we show that $\Gamma_{2} \cap B_{r} \subset \bar{Q}$ if $r$ is small. Let $\varrho>0$, then $u_{0}-p^{2}<0$ in $Q \backslash B_{\varrho}$, and therefore $u_{r}-p^{2}<0$ in $Q \backslash B_{\varrho}$ for small $r>0$. Hence $u<p^{2}$ in $Q \cap B_{r}$ for a small fixed $r>0$, and $\Gamma_{2} \cap Q \cap B_{r}=\emptyset$.

Now we can write $\Gamma_{2}=\Gamma_{2}^{+} \cup \Gamma_{2}^{-}$, where $\Gamma_{2}^{+} \cap \Gamma_{2}^{-}=\{0\}$, and $\Gamma_{2}^{ \pm}$are "squeezed" between $K$ and $Q$.

Step 2: We show that $\Gamma_{2}^{+}$is a $C^{1, \alpha}$-graph up to the origin. Fix any $x_{0} \in \Gamma_{2} \cap B_{r_{0} / 2}$, and denote by $d:=\left|x_{0}\right|$. Let $d_{0}:=\frac{d}{2} \sin \vartheta_{K}$, where $\vartheta_{K}$ is the opening angle of $K$. Since $x_{0} \notin K$ and 
$x_{0} \in Q$, we see that $B_{d_{0}}\left(x_{0}\right) \cap \mathcal{C}_{1}=\emptyset$ and $B_{d_{0}}\left(x_{0}\right) \cap \mathcal{S}_{2}=\emptyset$, see Figure 4.1. Hence the function $\omega:=p^{2}-u$ solves the following normalised obstacle problem

$$
\Delta \omega=\lambda_{2} \chi_{\{\omega>0\}} \text { in } B_{d_{0}}\left(x_{0}\right) .
$$

Now let us show that $p^{2}-u_{0}$ is a halfspace solution for (4.47). Denote by $\nu(0)$ the unit upward normal to the line $\left\{\theta=\phi_{1} / 2\right\}$, as indicated in Figure 4.1;

$$
\nu(0)=\left(-\sin \frac{\phi_{1}}{2}, \cos \frac{\phi_{1}}{2}\right) .
$$

The following is true,

$$
u_{0}(x)=p^{2}(x)-2(\nu(0) \cdot x)_{+}^{2} \quad \text { if } u_{0}(x)>p^{1}(x), \text { and } x \notin \mathcal{S}_{2} .
$$

Indeed, according to Lemma 3.7, if $x \in \mathcal{S}_{1}$, then

$$
\begin{array}{r}
p^{2}(x)-u_{0}(x)=x_{1}^{2}+x_{2}^{2}-x_{1}^{2} \cos \phi_{1}+x_{2}^{2} \cos \phi_{1}-2 x_{1} x_{2} \sin \phi_{1} \\
=2 x_{1}^{2} \sin ^{2} \frac{\phi_{1}}{2}+2 x_{2}^{2} \cos ^{2} \frac{\phi_{1}}{2}-4 x_{1} x_{2} \sin \frac{\phi_{1}}{2} \cos \frac{\phi_{1}}{2} \\
=2\left(-x_{1} \sin \frac{\phi_{1}}{2}+x_{2} \cos \frac{\phi_{1}}{2}\right)^{2}=2(\nu(0) \cdot x)^{2} .
\end{array}
$$

Since $u_{0}=p^{2}$ in $\mathcal{C}_{2}$, the proof of (4.48) is complete. Hence $p^{2}-u_{0}$ is a halfspace solution for the obstacle problem in $B_{d_{0}}\left(x_{0}\right)$, depending on the direction $\nu(0)$. Therefore we obtain

$$
\begin{array}{r}
\left\|\nabla_{\nu(0)}^{\prime} \omega\right\|_{L^{2}\left(B_{d_{0}}\left(x_{0}\right)\right)}=\left\|\nabla_{\nu(0)}^{\prime}\left(u-u_{0}-2(\nu(0) \cdot x)_{+}^{2}\right)\right\|_{L^{2}\left(B_{d_{0}}\left(x_{0}\right)\right)} \\
=\left\|\nabla_{\nu(0)}^{\prime}\left(u-u_{0}\right)\right\|_{L^{2}\left(B_{d_{0}}\left(x_{0}\right)\right)} \leq 2\left\|\nabla\left(u-u_{0}\right)\right\|_{L^{2}\left(B_{d_{0}}\left(x_{0}\right)\right)}
\end{array}
$$

where by definition $\nabla_{e}^{\prime}:=\nabla-e(\nabla \cdot e)$ for a unit vector $e$.

According to Lemma 4.1

$$
\left\|u-u_{0}\right\|_{W^{1,2}\left(B_{d_{0}}\left(x_{0}\right)\right)} \leq c\left\|u-u_{0}\right\|_{L^{2}\left(B_{2}\right)},
$$

hence by (4.49)

$$
\left\|\nabla_{\nu(0)}^{\prime} \omega\right\|_{L^{2}\left(B_{d_{0}}\left(x_{0}\right)\right)}=\left\|\nabla\left(u-u_{0}\right)\right\|_{L^{2}\left(B_{d_{0}}\left(x_{0}\right)\right)} \leq c\left\|u-u_{0}\right\|_{L^{2}\left(B_{2}\right)} \leq c \delta,
$$

which says that $\omega$ is almost flat in the direction $\nu(0)$. According to Theorem 8.1 in [2], $\Gamma_{2} \cap$ $B_{d_{0} / 2}\left(x_{0}\right)$ is a $C^{1, \gamma}$-graph, and there exists a unit normal vector to $\Gamma_{2}$ at the point $x_{0}$, denote it by $\nu\left(x_{0}\right)$. Furthermore, it follows from Corollary 8.1 in [2] and inequality (4.44) that

$$
\begin{array}{r}
\left|\nu\left(x_{0}\right)-\nu(0)\right| \leq c d_{0}^{-1}\left\|\nabla_{\nu(0)}^{\prime} \omega\right\|_{L^{2}\left(B_{d_{0}}\left(x_{0}\right)\right)} \leq c d_{0}^{-1}\left\|\nabla\left(u-u_{0}\right)\right\|_{L^{2}\left(B_{2 d}\right)} \\
=16 c d_{0}^{-1} d\left(\int_{B_{1 / 2}}\left|\nabla u(4 d y)-\nabla u_{0}(4 d y)\right|^{2} d y\right)^{\frac{1}{2}} \leq \\
\leq d_{0}^{-1} d\left\|u_{4 d}-u_{0}\right\|_{L^{2}\left(B_{1}\right)} \\
\leq \frac{c d}{d_{0}} d^{\gamma}\left\|u-u_{0}\right\|_{L^{2}\left(B_{2}\right)},
\end{array}
$$

where $c$ stands for a general constant, and it does not depend on $d$. Now we may conclude that

$$
\left|\nu\left(x_{0}\right)-\nu(0)\right| \leq \frac{c d}{d_{0}} d^{\gamma} \delta=\frac{c\left|x_{0}\right|^{\gamma}}{\sin \vartheta_{K}}\left\|u-u_{0}\right\|_{L^{2}\left(B_{2}\right)},
$$


and therefore $\Gamma_{2}^{+}$is a $C^{1, \gamma}$-graph up to the origin, for any $0<\gamma<1$.

The proof of $C^{1, \gamma}$-regularity for $\Gamma_{2}^{-}$can be obtained similarly. In that case $\Gamma_{2}^{-}$is almost flat in the direction $\nu_{2}^{-}(0)=\left(-\cos \frac{\phi_{2}}{2},-\sin \frac{\phi_{2}}{2}\right) \neq \nu(0)$, and we see that $\Gamma_{2}$ is Lipschitz, but it is not $C^{1}$ at the origin.

By a similar argument we can study $\Gamma_{1}$, and see that $\Gamma_{1}^{+}$is almost flat in the direction $\nu_{1}^{+}(0)=\left(\cos \frac{\phi_{1}}{2}, \sin \frac{\phi_{1}}{2}\right)$. Observe that $\nu_{1}^{+}(0)$ and $\nu(0)$ are orthogonal, which means $\Gamma_{1}^{+}$and $\Gamma_{2}^{+}$ cross at the origin.

It follows from energy characterisation of free boundary points, (4.4), and from Corollary 4.9 that if at a free boundary point $x_{0}$, the solution $u$ has a halfspace blow-up solution corresponding to $p^{i}$, then all possible blow-ups at $x_{0}$ are also halfspace solutions.

Theorem 4.11. Let $u$ solve the double obstacle problem in $B_{1}$ with obstacles $p^{1} \leq p^{2}$. If $u_{r_{j}} \rightarrow u^{1}$ as $j \rightarrow \infty$, where $u^{1}$ is a halfspace solution corresponding to $p^{1}$, then the blow-up of $u$ at the origin is unique, and $\Gamma_{1} \cap B_{1 / 2}$ is a $C^{1, \gamma}$-curve.

Proof. Without loss of generality we may assume that $u^{1}=p^{1}$ if $x_{2} \leq 0$. We can employ the same idea we used in Definiton 4.4, when defining minimal double-cone solutions, in order to define minimal halfspace solutions corresponding to $p^{1}$ : We say that $u^{1}$ is a minimal halfspace solution with respect to $u$, if it is closer to $u$ in $L^{2}\left(B_{1}\right)$-norm than any other halfspace solution. Employing a very similar flatness improvement argument, we obtain the uniqueness of blow-up limits, and the $C^{1, \gamma}$-regularity of $\Gamma_{1}$. Let us provide a brief sketch of the proof.

Let $\mu^{j}$ be a minimal halfspace solution with respect to $u^{j}:=u_{r_{j}}$, and let $v^{j}$ be the function defined in (4.20), then $v^{j} \rightarrow v^{0}$ weakly in $W^{1,2}\left(B_{1}\right)$ and strongly in $L^{2}\left(B_{1}\right)$, where $v^{0}$ is harmonic in the halfplane $\left\{x_{2}>0\right\}$, and vanishes on the halfplane $\left\{x_{2} \leq 0\right\}$, see the proof of Proposition 4.5. Hence $v^{0} \Delta v^{0}=0$ in a weak sense, and

$$
v^{0}(x)=v^{0}(r, \theta)=\sum_{k=1}^{\infty} A_{k} r^{k} \sin (k \theta) \text { in }\left\{x_{2}>0\right\} .
$$

By Lemma 4.2 $v^{j} \rightarrow v^{0}$ in $W^{1,2}\left(B_{1 / 2}\right)$, and therefore $A_{1}=0$. Furthermore, by the minimality assumption, $v^{0}$ is orthogonal to the function $r^{2} \sin (2 \theta)$, and therefore $A_{2}=0$, which implies that

$$
\left\|v^{0}(s x)\right\|_{L^{2}\left(B_{1}\right)} \leq s^{3}\left\|v^{0}\right\|_{L^{2}\left(B_{1}\right)},
$$

we refer to the proof of Lemma 4.6 for a similar argument. Repeating the iteration argument in Corollary 4.7 and Proposition 4.8 we will obtain that $\Gamma_{1}$ is a $C^{1, \gamma}$-curve in a neighbourhood of the origin. We leave out the details, since the technique is very similar to the argument we used earlier.

Let us observe that Theorem 4.11 on the uniqueness of halfspace blow-up limits holds also in Cases 2, 3, since we do not use any relation between obstacles in the proof. All we need is to know that we can rotate the free boundary, and choose a minimal halfspace solution, which provides the precious orthogonality property.

The following lemma from one variable calculus will be quite useful when showing the uniqueness of blow-ups in case there are only finitely many blow-up solutions.

Lemma 4.12. Let $f$ be a nonnegative continuous function in the interval $(0,1)$, and assume that

$$
\liminf _{t \rightarrow 0+} f(t)=0 \text { and } \limsup _{t \rightarrow 0+} f(t)=A>0 .
$$


Then for any $0<a<A$ there exists a sequence $t_{j} \rightarrow 0$, such that $f\left(t_{j}\right) \rightarrow a$.

Proof. Fix $0<a<A$, and let $y_{j} \rightarrow 0+$ and $z_{j} \rightarrow 0+$ be such that $\left|f\left(y_{j}\right)-A\right| \leq \frac{1}{j}$ and $0 \leq f\left(z_{j}\right) \leq \frac{1}{j}$. Taking $j$ large enough, we insure that $f\left(y_{j}\right)>a$ and $f\left(z_{j}\right)<a$. Since $f$ is a continuous function, by intermediate value theorem there exists $t_{j} \rightarrow 0+$, such that $f\left(t_{j}\right)=a$.

Corollary 4.13. Let $u$ be the solution to the double obstacle problem with polynomial obstacles $p^{1} \leq p^{2}$. If $u_{r_{j}} \rightarrow p^{i}$ through a subsequence, then the blow-up of $u$ at the origin is unique.

Proof. Let $f(r):=\left\|u_{r}-p^{1}\right\|_{L^{2}\left(B_{1}\right)}$. If there exists a sequence $s_{j} \rightarrow 0+$, such that $u_{s_{j}} \rightarrow p^{2}$. Then the function $f$ satisfies the assumptions in Lemma 4.12 but $f$ has only two limit points. Therefore there exists $\lim _{r \rightarrow 0+} f(r)=0$.

\section{$5 \quad$ Uniqueness of blow-ups, Case 2}

The uniqueness of blow-ups in Case 2) follows from Theorem 3.8 and from Lemma 4.12 ,

Theorem 5.1. Let $u$ be a solution to the double obstacle problem with obstacles

$$
p^{1}(x)=a_{1} x_{1}^{2}+c_{1} x_{2}^{2} \text { and } p^{2}(x)=a_{2} x_{1}^{2}+c_{2} x_{2}^{2},
$$

satisfying (3.9) and (3.8) and assume that $\left(a_{1}+c_{2}\right)\left(c_{1}+a_{2}\right)<0$. If $u_{r_{j}} \rightarrow \mu$ for a subsequence $r_{j} \rightarrow 0+$, where $\mu$ is a double-cone solution, then u has a unique blow-up at the origin;

$$
\frac{u(r x)}{r^{2}} \rightarrow \mu(x) .
$$

Proof. The proof follows from Lemma 4.12. Assume that $u_{r_{j}} \rightarrow \mu$ for a subsequence $r_{j} \rightarrow 0+$, and denote by $f(r):=\left\|u_{r}-\mu\right\|_{L^{2}\left(B_{1}\right)}$. If there exists a sequence $s_{j} \rightarrow 0+$, such that $u_{s_{j}} \rightarrow \mu_{1}$, and $\mu_{1} \neq \mu$. Then the function $f$ satisfies the assumptions in Lemma 4.12, but according to Theorem $3.8 f$ has only four limit points. Therefore there exists $\lim _{r \rightarrow 0+} f(r)=0$.

Now let us discuss the speed of the convergence $\left\|u_{r}-\mu\right\|_{L^{2}\left(B_{1}\right)} \rightarrow 0$ as $r \rightarrow 0$, and the regularity of the free boundary.

Remark 5.2. Let $u$ be a solution to the double obstacle problem in Case 2, and assume that $u_{r_{j}} \rightarrow \mu$, where $\mu$ is a double-cone solution. Arguing as we did in the proof of Theorem 4.10, and employing Theorem [5.1, we can show that the free boundary is a union of four $C^{1}$-curves. Although we do not have a uniform estimate like (4.50) yet.

With a modification of our flatness improvement argument, we can show uniform $C^{1, \gamma_{-}}$ regularity of $\Gamma_{i}$ up to the origin also in Case 2.

Theorem 5.3. Let $u$ be a solution to the double obstacle problem in $B_{1}$ with obstacles $p^{i}(x)=$ $a_{i} x_{1}^{2}+c_{i} x_{2}^{2}$, satisfying (3.9) and (3.8) and assume that $\left(a_{1}+c_{2}\right)\left(c_{1}+a_{2}\right)<0$. Let $u_{r} \rightarrow \mu$, where $\mu$ is a double-cone solution. Denote by $\vartheta$ the opening angle of $\mathcal{S}_{i}$. Then

$$
\left\|u_{r}-\mu\right\|_{L^{2}\left(B_{1}\right)} \leq C r^{\gamma}\|u-\mu\|_{L^{2}\left(B_{2}\right)},
$$

where $0<\gamma<1$ depends on $\vartheta$. Furthermore, the free boundary $\Gamma_{u}$ is a union of four $C^{1, \gamma}$-curves. 
Proof. We provide only a brief sketch of the proof, since the detailed proof would be quite long, and very similar to the proofs of Proposition 4.8 and of Theorem 4.10. Therefore we focus on the main differences of double-cone solutions in Cases 1 and 2. Let

$$
v_{r}:=\frac{u_{r}-\mu}{\left\|u_{r}-\mu\right\|_{L^{2}\left(B_{2}\right)}}
$$

where $\mu$ is a double-cone solution. Then $v_{r} \rightarrow v^{0}$ through a subsequence $r_{j} \rightarrow 0+$ weakly in $W^{1,2}\left(B_{1}\right)$ and strongly in $L^{2}\left(B_{1}\right)$. Here $\Delta v^{0}=0$ in $\mathcal{S}_{i}$, and $v^{0}=0$ in $\mathbb{R}^{2} \backslash\left(\mathcal{S}_{1} \cup \mathcal{S}_{2}\right)$, see the proof of Proposition 4.5. Hence

$$
v^{0}(r, \theta)=\sum_{k=1}^{\infty} r^{\alpha_{k}}\left(A_{k}^{i} \cos \alpha_{k} \theta+B_{k}^{i} \sin \alpha_{k} \theta\right) \text { in } \mathcal{S}_{i}, i=1,2
$$

where $\alpha_{k}=\frac{\pi k}{\vartheta}$ and $\vartheta$ is the opening angle of the cone $\mathcal{S}_{i}$ for $i=1,2$. According to Theorem 3.8 , $0<\cos ^{2} \vartheta<1$, hence there are two possible cases; either $\left.i\right) 0<\vartheta<\pi / 2$ or $\left.i i\right) \pi / 2<\vartheta<\pi$. We discuss these two cases separately.

i) $0<\vartheta<\pi / 2$, then $\alpha_{k}=\pi k / \vartheta>2 k$, for all $k=1,2, \ldots$. It follows that

$$
\left\|v_{s}^{0}\right\|_{L^{2}\left(B_{1}\right)} \leq s^{\kappa}\left\|v_{0}\right\|_{L^{2}\left(B_{1}\right)},
$$

where $\kappa=\pi / \vartheta-2$, and a standard iteration argument leads to (5.2) with $0<\gamma<\kappa$.

ii) $\pi / 2<\vartheta<\pi$, then $1<\alpha_{1}=\pi / \vartheta<2$ and $\alpha_{2}=2 \pi / \vartheta>2$. We will obtain (5.5) with $\kappa=2 \pi / \vartheta-2$, if we show that $A_{1}^{i}=B_{1}^{i}=0$ in (5.4). Assume not, i.e. if $\left|A_{1}^{1}\right|+\left|B_{1}^{1}\right|>0$, then $\left\|v_{s}^{0}\right\|_{L^{2}\left(B_{1}\right)} \geq c s^{\alpha_{1}-2}$, where $c>0$ is a fixed constant depending on $A_{1}^{1}, B_{1}^{1}$ and $0<s<1$ is any number. Since $v^{j}:=v_{r_{j}} \rightarrow v^{0}$ in $L^{2}\left(B_{1}\right)$, then for any fixed $1>\tau>s>0$ there exist $\varepsilon>0$ small, such that

$$
\left\|u_{s}-\mu\right\|_{L^{2}\left(B_{1}\right)} \geq c \tau^{\alpha_{1}-2}\|u-\mu\|_{L^{2}\left(B_{2}\right)},
$$

provided $\|u-\mu\|_{L^{2}\left(B_{2}\right)} \leq \varepsilon$. Indeed, if the last statement is not true, then there exist $1>\tau>$ $s>0$, and a sequence $u^{j}$, such that $\left\|u^{j}-\mu\right\|_{L^{2}\left(B_{2}\right)} \leq \varepsilon_{j} \rightarrow 0+$, but

$$
\frac{\left\|u_{s}^{j}-\mu\right\|_{L^{2}\left(B_{1}\right)}}{\left\|u^{j}-\mu\right\|_{L^{2}\left(B_{2}\right)}}<c \tau^{\alpha_{1}-2} .
$$

After passing to the limit as $j \rightarrow \infty$ in (5.7), we obtain $\left\|v_{s}^{0}\right\|_{L^{2}\left(B_{1}\right)} \leq c \tau^{\alpha_{1}-2}$, contradicting $\left\|v_{s}^{0}\right\|_{L^{2}\left(B_{1}\right)} \geq c s^{\alpha_{1}-2}$.

According to Theorem 5.1 for any $\varepsilon>0$ small there exists $k_{\varepsilon}$ such that $\left\|u_{s^{k}}-\mu\right\|_{L^{2}\left(B_{2}\right)} \leq \varepsilon$ for all $k \geq k_{\varepsilon}$. The latter together with (5.6) implies that for any positive integer $m$

$$
\begin{array}{r}
\varepsilon \geq\left\|u_{s^{k+m}}-\mu\right\|_{L^{2}\left(B_{2}\right)} \geq c \tau^{\alpha_{1}-2}\left\|u_{s^{k+m-1}}-\mu\right\|_{L^{2}\left(B_{2}\right)} \\
\geq\left(\tau^{\alpha_{1}-2} c\right)^{2}\left\|u_{s^{k+m-2}}-\mu\right\|_{L^{2}\left(B_{2}\right)} \geq \ldots \geq\left(\tau^{\alpha_{1}-2} c\right)^{m}\left\|u_{s^{k_{\varepsilon}}}-\mu\right\|_{L^{2}\left(B_{2}\right)} .
\end{array}
$$

Recalling that $\alpha_{1}<2$, and taking $1>\tau>s>0$ small, we obtain a contradiction, when letting $m \rightarrow \infty$. Hence $\left|A_{1}^{i}\right|=\left|B_{1}^{i}\right|=0$, and (5.5) holds with $\kappa=\alpha_{2}-2$. An iteration argument similar to the one used in Corollary 4.7 and Proposition 4.8 leads to the following inequalities, $\left\|u_{s^{k}}-\mu\right\|_{L^{2}\left(B_{1}\right)} \leq \tau^{k \gamma}\|u-\mu\|_{L^{2}\left(B_{2}\right)}$, whith $1>\tau>s$ and $\left\|u_{r}-\mu\right\|_{L^{2}\left(B_{1}\right)} \leq C r^{\gamma}\|u-\mu\|_{L^{2}\left(B_{2}\right)}$, where $0<\gamma<\alpha_{2}-2$, and $0<r<1$ small. Applying the proof of Theorem 4.10, we deduce that $\Gamma_{i}$ is Lipschitz and consists of two $C^{1, \gamma}$-curves, meeting at the origin. 
Now let us briefly discuss the uniqueness of halfspace blow-up limits. Although in Case 2, the lines $\Gamma_{i}$ are not rotationally invariant on the plane, but they are rotationally invariant inside a fixed cone, depending on the given obstacles. Hence we can define minimal halfspace solutions in this case as well, and obtain the uniqueness of blow-ups (see Theorem 4.11).

\section{Uniqueness of blow-ups, Case 3}

Consider the double obstacle problem with obstacles

$$
p^{1}(x)=a_{1} x_{1}^{2}+c_{1} x_{2}^{2} \text { and } p^{2}(x)=a_{2} x_{1}^{2}+c_{2} x_{2}^{2},
$$

satisfying (3.8), (3.9), and let $P$ be the polynomial in (3.31).

In Case 3, the polynomial $P$ has a sign, and according to Theorems 3.8 and 3.11 we have only halfspace solutions. Without loss of generality we may assume that $P \geq 0$. According to Theorem 3.11 there are infinitely many rotational invariant halfspace solutions corresponding to $p^{1}$, and we can apply our flatness improvement argument (Theorem 4.11) in order to show the uniqueness of blow-ups and $C^{1, \gamma}$-regularity of $\Gamma_{1}$.

\section{$7 \quad$ An example of a double-cone solution in $\mathbb{R}^{3}$}

Let $u_{0}$ be a homogeneous global solution to the double obstacle problem with obstacles $p^{1} \leq p^{2}$ in $\mathbb{R}^{3}$. As usually we assume that the origin is a free boundary point, $p^{1}(0)=p^{2}(0)=0$, and we want to understand the behaviour of the free boundary at the origin. We split the discussion into three cases.

If $p^{1}=p^{2}$ on a plane, then we obtain only halfspace solutions. If $p^{1}=p^{2}$ on a line, then we can analyse the possible blow-up solutions, based on our results obtained in dimension $n=2$. In particular, we can see that in this case there are no three-dimensional double-cone solutions. The proofs of the last statements can be obtained via a dimension reduction technique. However, we omit the proofs, since our aim is to find a three-dimensional double-cone solution.

Our knowledge on the existence of double-cone solutions in dimension two suggest that we may obtain three-dimensional double-cone solutions, assuming that $p^{1}$ and $p^{2}$ meet only at a single point. However, since in dimension $n=3$ homogeneous degree two harmonic functions are not necessarily polynomials, the analysis is much more complicated. In this section we give an example of a three-dimensional double-cone solution, symmetric with respect to the $z$-axes and the $(x, y)$-plane.

\subsection{Solutions symmetric with respect to the $z$-axes}

Let $p^{1} \leq p^{2}$ be given homogeneous degree two polynomials, meeting only at the origin. We are looking for two closed cones $\mathcal{C}_{1}, \mathcal{C}_{2}$ and for a harmonic homogeneous degree two function $q$ in $\mathbb{R}^{3} \backslash\left(\mathcal{C}_{1} \cup \mathcal{C}_{2}\right)$, such that

$$
\Delta q=0, p^{1}<q<p^{2} \text { in } \mathbb{R}^{3} \backslash\left(\mathcal{C}_{1} \cup \mathcal{C}_{2}\right)
$$

and

$$
q-p^{1}=\left|\nabla q-\nabla p^{1}\right|=0 \text { on } \partial \mathcal{C}_{1}, \text { and } q-p^{2}=\left|\nabla q-\nabla p^{2}\right|=0 \text { on } \partial \mathcal{C}_{2}
$$


Let $(r, \phi, \theta)$ represent the spherical coordinates in $\mathbb{R}^{3}$,

$$
x=r \cos \phi \sin \theta, y=r \sin \phi \sin \theta, z=r \cos \theta, \text { where } r \geq 0,0 \leq \phi<2 \pi, 0 \leq \theta \leq \pi,
$$

then $q(r, \phi, \theta)=r^{2} \zeta(\phi, \theta)$ by homogenuity. Furthermore, assume that $q$ is symmetric with respect to the $z$-axes, i.e. $\zeta(\phi, \theta)=\zeta(\theta)$. Using the expression for the Laplace operator in spherical coordinates, we obtain the following ordinary differential equation for $\zeta$,

$$
\zeta^{\prime \prime}+\frac{\cos \theta}{\sin \theta} \zeta^{\prime}+6 \zeta=0
$$

We can see via a substitution that $\zeta_{1}=1+3 \cos 2 \theta$ is a solution to (7.3). Using reduction of order, we get another solution, $\zeta_{2}=3 \cos \theta+\frac{1+3 \cos 2 \theta}{4} \ln \frac{1-\cos \theta}{1+\cos \theta}$. Thus the general solution to (7.3) is given by

$$
\zeta=A(1+3 \cos 2 \theta)+B\left(3 \cos \theta+\frac{1+3 \cos 2 \theta}{4} \ln \frac{1-\cos \theta}{1+\cos \theta}\right),
$$

where $A, B$ are real numbers. Hence

$$
q=A r^{2}(1+3 \cos 2 \theta)+B r^{2}\left(3 \cos \theta+\frac{1+3 \cos 2 \theta}{4} \ln \frac{1-\cos \theta}{1+\cos \theta}\right) .
$$

We are looking for a solution to the double obstacle problem, symmetric with respect to the $z$-axes. Hence we assume that the obstacles $p^{1}$ and $p^{2}$ are so, i. e. they do not depend on $\phi$. Let $t:=\cos \theta, t \in(-1,1)$, and take

$$
p^{1}=\left(a_{1}+b_{1} t^{2}\right) r^{2}, \quad p^{2}=\left(a_{2}+b_{2} t^{2}\right) r^{2}, \quad \text { where } a_{1}<a_{2}, a_{2}-a_{1}+b_{2}-b_{1}>0,
$$

then $p^{1} \leq p^{2}, p^{1}$ and $p^{2}$ meet only at the origin. Also, observe that

$$
q=A r^{2}\left(3 t^{2}-1\right)+B r^{2}\left(3 t+\frac{3 t^{2}-1}{2} \ln \frac{1-t}{1+t}\right),
$$

for some $A, B$. In order to construct an example of a double-cone solution, we want to find $A, B, a_{i}, b_{i}$ and $1>t_{1}>t_{2}>-1$, such that

$$
\begin{array}{r}
f_{1}(t):=\frac{q-p^{1}}{r^{2}}=A\left(3 t^{2}-1\right)-a_{1}-b_{1} t^{2}+B\left(3 t+\frac{3 t^{2}-1}{2} \ln \frac{1-t}{1+t}\right) \geq 0, \\
f_{2}(t):=\frac{p^{2}-q}{r^{2}}=a_{2}+b_{2} t^{2}-A\left(3 t^{2}-1\right)-B\left(3 t+\frac{3 t^{2}-1}{2} \ln \frac{1-t}{1+t}\right) \geq 0, \text { for } t_{2} \leq t \leq t_{1},
\end{array}
$$

and

$$
f_{1}\left(t_{1}\right)=f_{1}^{\prime}\left(t_{1}\right)=0, \quad f_{2}\left(t_{2}\right)=f_{2}^{\prime}\left(t_{2}\right)=0 .
$$

Let us rewrite

$$
\begin{array}{r}
f_{1}(t)=t^{2}\left(3 A-b_{1}\right)-a_{1}-A+B\left(3 t+\frac{3 t^{2}-1}{2} \ln \frac{1-t}{1+t}\right), \text { and } \\
f_{2}(t)=t^{2}\left(-3 A+b_{2}\right)+a_{2}+A-B\left(3 t+\frac{3 t^{2}-1}{2} \ln \frac{1-t}{1+t}\right) .
\end{array}
$$

Denote by

$$
g(t):=3 t+\frac{3 t^{2}-1}{2} \ln \frac{1-t}{1+t}, \text { for } t \in(-1,1),
$$


and observe that $g$ is an odd function. From (7.7) we obtain the following system;

$$
\left\{\begin{array}{l}
f_{1}\left(t_{1}\right)=t_{1}^{2}\left(3 A-b_{1}\right)-a_{1}-A+B g\left(t_{1}\right)=0 \\
f_{1}^{\prime}\left(t_{1}\right)=2 t_{1}\left(3 A-b_{1}\right)+B g^{\prime}\left(t_{1}\right)=0 \\
f_{2}\left(t_{2}\right)=t_{2}^{2}\left(-3 A+b_{2}\right)+a_{2}+A-B g\left(t_{2}\right)=0 \\
f_{2}^{\prime}\left(t_{2}\right)=2 t_{2}\left(-3 A+b_{2}\right)-B g^{\prime}\left(t_{2}\right)=0
\end{array}\right.
$$

In order to simplify the case further, we assume that the free boundary of the desired double-cone solution is symmetric with respect to the $(x, y)$-plane. Hence $t_{1}=-t_{2}=t_{0} \neq 0$, and

$$
\left\{\begin{array}{l}
t_{0}^{2}\left(3 A-b_{1}\right)-a_{1}-A+B g\left(t_{0}\right)=0 \\
2 t_{0}\left(3 A-b_{1}\right)+B g^{\prime}\left(t_{0}\right)=0 \\
t_{0}^{2}\left(-3 A+b_{2}\right)+a_{2}+A+B g\left(t_{0}\right)=0 \\
-2 t_{0}\left(-3 A+b_{2}\right)-B g^{\prime}\left(t_{0}\right)=0
\end{array}\right.
$$

where we used that $g$ is an odd function, and $g^{\prime}$ is an even function. The system (7.10) is equivalent to the following system

$$
\left\{\begin{array}{l}
2 A+a_{1}+a_{2}=0 \\
6 A-b_{1}-b_{2}=0 \\
t_{0}^{2}\left(b_{2}-b_{1}\right)+a_{2}-a_{1}+2 B g\left(t_{0}\right)=0 \\
t_{0}\left(b_{2}-b_{1}\right)+B g^{\prime}\left(t_{0}\right)=0
\end{array}\right.
$$

Now let us take $b_{1}=b_{2}=b$, then $3 A-b_{1}=-3 A+b_{2}=0$ and

$$
f_{1}(t)=-a_{1}-A+B g(t)=\frac{a_{2}-a_{1}}{2}+B g(t), f_{2}(t)=a_{2}+A-B g(t)=\frac{a_{2}-a_{1}}{2}-B g(t) .
$$

On the other hand, the assumption $b_{1}=b_{2}$ implies that $B g^{\prime}\left(t_{0}\right)=0$, and $a_{2}-a_{1}=-2 B g\left(t_{0}\right)>0$, hence $B \neq 0$, and $g^{\prime}\left(t_{0}\right)=0$. It is easy to verify that $t_{0}$ is unique in the interval $(0,1), g\left(t_{0}\right)>0$, and $g^{\prime}(t) \geq 0$, for $-t_{0} \leq t \leq t_{0}$. Hence $g$ is a monotone increasing function in the interval $\left(-t_{0}, t_{0}\right)$, and therefore

$$
\begin{aligned}
f_{1}(t) & =\frac{a_{2}-a_{1}}{2 g\left(t_{0}\right)}\left(g\left(t_{0}\right)-g(t)\right) \geq 0 \text { and } \\
f_{2}(t)=\frac{a_{2}-a_{1}}{2 g\left(t_{0}\right)}\left(g\left(t_{0}\right)+g(t)\right) & =\frac{a_{2}-a_{1}}{2 g\left(t_{0}\right)}\left(-g\left(-t_{0}\right)+g(t)\right) \geq 0 .
\end{aligned}
$$

Now we can write an explicit example.

Example 7.1. Let

$$
p^{1}(r, \phi, \theta)=-r^{2}, p^{2}(r, \phi, \theta)=r^{2},
$$

in spherical coordinates, and

$$
q(r, \phi, \theta)=-\frac{r^{2} g(\cos \theta)}{g\left(t_{0}\right)},
$$

where $g(t)=3 t+\frac{3 t^{2}-1}{2} \ln \frac{1-t}{1+t}$ is defined for $t \in(-1,1)$, and $0<t_{0}<1$ is chosen so that $g^{\prime}\left(t_{0}\right)=0$. Then

$$
u= \begin{cases}p^{1} & \text { if } 0 \leq \theta \leq \arccos \left(t_{0}\right) \\ q & \text { if } \arccos \left(t_{0}\right) \leq \theta \leq \pi-\arccos \left(t_{0}\right) \\ p^{2} & \text { if } \pi-\arccos \left(t_{0}\right) \leq \theta \leq \pi\end{cases}
$$

is a double-cone solution to the double obstacle problem with obstacles $p^{1}, p^{2}$. 
Proof. Let $p^{1}=-r^{2}$ and $p^{2}=r^{2}$, then $a_{1}=-a_{2}=1$ and $b_{1}=b_{2}=0$ in (7.5). Hence we obtain from (7.11) that $A=0, B=-1 / g\left(t_{0}\right)$, and $q=-r^{2} \frac{g(\cos \theta)}{g\left(t_{0}\right)}$. Denote by

$$
\mathcal{C}_{1}:=\left\{(r, \phi, \theta): 0 \leq \theta \leq \arccos \left(t_{0}\right)\right\} \text { and } \mathcal{C}_{2}:=\left\{(r, \phi, \theta): \pi-\arccos \left(t_{0}\right) \leq \theta \leq \pi\right\} .
$$

Then $q(r, \phi, \theta)$ is a harmonic function in $\mathbb{R}^{3} \backslash\left(\mathcal{C}_{1} \cup \mathcal{C}_{2}\right)$, satisfying 17.1$)$ and the boundary conditions (7.2). Hence the function $u$, defined in (7.13), is a homogeneous global solution to the double obstacle problem with obstacles $p^{1}, p^{2}$. The coincidence sets $\left\{u=p^{1}\right\}=\mathcal{C}_{1}$ and $\left\{u=p^{2}\right\}=\mathcal{C}_{2}$ are cones with a common vertex, thus $u$ is a double-cone solution.

It follows from our classification of blow-up solutions in $\mathbb{R}^{2}$ and from Example 7.1, that in $\mathbb{R}^{3}$ there are at least four types of blow-ups; polynomial, halfspace, double-cone solutions, and solutions, for which the free boundary is a union of four halfplanes. The complete analysis of homogeneous global solutions and the regularity of the free boundary for the double obstacle problem in dimension $n=3$ we leave for a future publication.

\section{References}

[1] Gohar Aleksanyan. Optimal regularity in the optimal switching problem. Ann. Inst. H. Poincaré Anal. Non Linéaire 33 (2016), no. 6, 1455-1471.

[2] John Andersson. The obstacle problem. 2016.

[3] John Andersson, Henrik Shahgholian, and Georg S. Weiss. Double obstacle problems with obstacles given by non- $C^{2}$ Hamilton-Jacobi equations. Arch. Ration. Mech. Anal. 206, no. 3, 779-819., 2012.

[4] L. A. Caffarelli. The obstacle problem revisited. J. Fourier Anal. Appl. 4 (1998), no. 4-5, 383-402.

[5] Alessio Figalli and Henrik Shahgholian. An overview of unconstrained free boundary problems. Philos. Trans. A 373 (2015), no. 2050, 20140281, 11 pp.

[6] Ki-Ahm Lee, Jinwan Park, and Henrik Shahgholian. The regularity theory for the double obstacle problem. arxiv:1703.06262v1 